\title{
Vertical emittance reduction and preservation in electron storage rings via resonance driving terms correction
}

\author{
A. Franchi, L. Farvacque, J. Chavanne, F. Ewald, B. Nash, and K. Scheidt \\ ESRF, Grenoble, France \\ R. Tomás \\ CERN, Geneva, Switzerland \\ (Received 29 November 2010; published 25 March 2011)
}

\begin{abstract}
In this paper the influence of betatron coupling on the transverse beam emittances is described using the resonance driving terms formalism. Betatron coupling and vertical dispersion generated by magnetic and installation errors are major sources of vertical emittance. A new scheme for minimizing the latter is presented here, together with results from measurements carried out in 2010 at the ESRF electron storage ring, which provided vertical emittance of about $4.4 \mathrm{pm}$, a record low for this machine. Two schemes for the automatic compensation of coupling introduced by insertion devices are also presented with results from the first implementation tests. This paper is also an attempt to clarify the various definitions and meanings of vertical emittance in the presence of coupling.
\end{abstract}

DOI: 10.1103/PhysRevSTAB.14.034002

PACS numbers: $29.20 .-\mathrm{c}$

\section{INTRODUCTION}

Third-generation light sources and modern lepton colliders are characterized by low transverse beam emittances (below $10 \mathrm{~nm}$ and $20 \mathrm{pm}$ for the horizontal and the vertical plane, respectively). Lepton colliders benefit from low vertical emittance, as their luminosity scales with the inverse of its square root. The interest of coupling correction for third-generation light sources is threefold. First, smaller vertical beam sizes allow the installation of vacuum chambers of ever smaller vertical aperture in straight sections housing insertion devices (IDs), making it possible to reach higher magnetic fields, and hence photon flux, by further reducing the gap between the two ID poles. Second, any reduction of the vertical emittance induces higher photon brilliance, provided that this is not yet limited by energy spread. Third, large horizontal oscillations during top-up injection experienced by the incoming off-axis beamlet would have limited impact on the vertical clearance. It is however worthwhile noticing that the achievement of ultralow vertical emittances generally lead to an important reduction of the Touscheck lifetime because of the reduced bunch volume. Ultralow vertical emittances were already obtained and directly measured in Ref. [1] (3.2 pm) and in Ref. [2] (1.7 pm). Values of $1.3 \mathrm{pm}$ have been recently reported in Ref. [3].

Because of radiation damping and diffusion, accelerator lattices define specific equilibrium (or eigen)emittances $\left(\mathcal{E}_{u}, \mathcal{E}_{v}\right)$ that are constant in time and along the ring. In

Published by American Physical Society under the terms of the Creative Commons Attribution 3.0 License. Further distribution of this work must maintain attribution to the author(s) and the published article's title, journal citation, and DOI. the absence of magnet errors, the two planes are decoupled and vertical dispersion $D_{y}$ is everywhere zero. This in turn leads to zero ideal vertical eigenemittance, $\mathcal{E}_{v}=0$. The impact of scattering effects, with residual gas as well as intrabeam collisions, is not taken into account here. Beam profile monitors provide root-mean-square (RMS) beam sizes, $\sigma_{r}^{2}=\left\langle r^{2}\right\rangle-\left(\delta D_{r}\right)^{2}$, where $r$ stands for either $x$ or $y$, $D_{r}$ is the dispersion function, and $\delta$ is the relative RMS beam energy spread. Throughout the paper, all RMS quantities denoted with $\sigma$ refer to the betatronic part, hence assuming that dispersion terms have been already subtracted. In the ideal uncoupled lattice, the eigenemittance $\mathcal{E}$ is equivalent to the RMS beam emittance $\epsilon$, defined as $\boldsymbol{\epsilon}_{r}=\sqrt{\sigma_{r} \sigma_{p}-\sigma_{r p}^{2}}=$ const, where $\sigma_{p}$ and $\sigma_{r p}$ are the RMS beam divergence and cross-term moment, respectively. The stored beam being matched to the focusing lattice, the measured profile is sufficient to characterize the RMS emittance, since

$$
\epsilon_{r}=\frac{\sigma_{r}^{2}}{\beta_{r}}=\frac{\left\langle r^{2}\right\rangle-\left(\delta D_{r}\right)^{2}}{\beta_{r}},
$$

where $\beta_{r}$ is the Twiss parameter.

Betatron coupling between the two transverse planes has in this context four main effects: (i) The vertical equilibrium emittance is no longer zero, $\mathcal{E}_{v} \neq 0$; (ii) RMS emittance and eigenemittance are no longer equivalent, $\epsilon_{r} \neq \mathcal{E}_{r}$; (iii) Eq. (1) is no longer valid, $\epsilon_{r} \neq \sigma_{r}^{2} / \beta_{r}$; (iv) the RMS emittance varies along the ring, i.e., depends on the coordinate $s, \boldsymbol{\epsilon}_{r}=\boldsymbol{\epsilon}_{r}(s)$. The literature related to this topic is already vast, both in terms of mathematical modeling and practical cures aimed at minimizing coupling effects. Regarding mathematical modeling of the beam equilibrium distribution, the first analytical formulas for 
the ideal uncoupled case date back to 1970 [4]. A numerical algorithm for the general coupled case was then proposed in 1979 [5]. In 1994 another general coupled analysis was carried out in Ref. [6], predicting the dependence of $\epsilon_{r}$ on $s$. In 1996 a pioneering work on the coupling resonance driving terms (RDTs) close to the difference resonance [7] indicated that the single-particle emittances (i.e. the horizontal and vertical actions) would oscillate along the ring instead of being constant. However, no conclusion was drawn on the RMS emittances. In 2006 even more general formulas for the equilibrium beam distribution were derived and applied to coupling (both betatron and synchrobetatron) and intrabeam scattering [8-10]: This formalism is used in the present paper to connect the RDTs to the equilibrium eigenemittances. Formulas for the numerical evaluation of the equilibrium beam distribution parameters of Refs. [5,6] were already implemented in optics code such as Accelerator Toolbox (AT) [11] and MADX [12].

Works on coupling correction and vertical emittance minimization [13-15] are based on beam size measurements at one or few locations. In Ref. [15] it is acknowledged that measurements of $\sigma_{y}^{2} / \beta_{y}$ are only an approximation of $\epsilon_{y}$ and both differ from the vertical eigenemittance $\mathcal{E}_{v}$ obtained from the model. Nevertheless, no direct estimation of such an approximation is made.

In this paper analytical and handy formulas will be derived relating the three quantities $\sigma_{r}^{2} / \beta_{r}, \epsilon_{r}$, and $\mathcal{E}_{r}$. It will be described how to evaluate $\epsilon_{r}$ and $\mathcal{E}_{r}$ from beam profile and RDT measurements. It will also explain why vertical emittance inferred from beam profile measurements at one or few locations around the ring may largely underestimate or overestimate $\epsilon_{y}$ in relative terms, inducing an uncertainty much larger than the typical experimental errors. Results for the damping and diffusion coefficients (and hence equilibrium emittances) already derived in Ref. [8] are here rewritten in the RDT formalism.

The RDT formalism of Refs. [16-19] provides a quantitative description of coupling effects in terms of the ideal lattice parameters (Twiss parameters and dispersion functions) and the magnet errors (modeled as localized skew quadrupole integrated strengths). This is done through the two coupling RDTs $f_{1001}$ and $f_{1010}$ of Refs. [17,20]. Both are measurable and their knowledge provides a direct evaluation of both the real RMS and the equilibrium emittance (in absence of synchrobetatron coupling). RDT correction results in the minimization of the vertical emittance. In Ref. [20] the focus was given to hadron beams near the difference resonance, where the emittance evolution is defined by both the coupling RDT $f_{1001}$ and the initial emittances $\epsilon_{r 0}$. In lepton machines with radiation damping and diffusion, the role of initial emittance is somehow replaced by the equilibrium emittances, that depend on the RDTs too.
At the ESRF storage ring coupling RDTs are inferred from orbit response matrix (ORM) measurement. A singlevalue-decomposition (SVD) scheme was recently developed to power 32 independent skew quadrupoles to minimize both vertical dispersion and coupling RDTs around the ring. The corrector setting so found, once implemented in the machine, yielded the lowest vertical emittance ever observed in this machine. In light sources this baseline correction may not last during beam delivery because of continuous changes in the ID vertical apertures performed by users. Magnetic imperfections, including skew quadrupole terms, are enhanced whenever the ID vertical aperture is reduced to its minimum $(6 \mathrm{~mm}$ in the case of in-vacuum undulators at the ESRF). Two schemes have been recently tested and implemented to automatically compensate for these errors.

The paper is structured as follows. In Sec. II the main explicit formulas relating the emittances to the lattice parameters and the coupling RDTs are presented and discussed, leaving all mathematical derivations in separate appendices. The RDT modeling based on orbit response matrix measurements is discussed in Sec. III, whereas the proposed scheme for coupling correction is examined in Sec. IV. Results from measurements and corrections carried out at the ESRF storage ring at constant ID vertical apertures in the first half of 2010 are presented in Sec. V. Principles and experimental results of the emittance preservation against variations of the ID vertical gaps are eventually reported in Sec. VI.

\section{COUPLING RDTS AND TRANSVERSE EMITTANCES}

With coupling the usual notion of emittance is replaced by three separate quantities. For the sake of clarity, it is worthwhile distinguishing the emittance nomenclature before analyzing their dependence of the RDTs. (i) The RMS apparent emittance is the observable quantity, derived from beam profile measurements

$$
\mathbb{E}_{r}(s)=\frac{\sigma_{r}^{2}(s)}{\beta_{r}(s)}=\frac{\left\langle r^{2}(s)\right\rangle-\left(\delta D_{r}(s)\right)^{2}}{\beta_{r}(s)} .
$$

(ii) The RMS projected emittance is the second-order statistical moment representing the surface of the beam phase space $\left(r, p_{r}\right)$,

$$
\epsilon_{r}(s)=\sqrt{\sigma_{r}(s) \sigma_{p}(s)-\sigma_{r p}^{2}(s)} .
$$

(iii) The eigenemittance or equilibrium emittance $\mathcal{E}$ is the true invariant (i.e. independent of $s$ ) and an intrinsic property of the accelerator lattice. In Appendix A the RDT formalism is applied to the single-particle betatron motion in the presence of coupling. The results of major interest in the context of this paper are here summarized. 


\section{A. RMS apparent emittances}

As far as the apparent emittances of Eq. (2) are concerned, the following relations apply (a dependence on $s$ has to be assumed in all quantities, bar the two eigenemittances $\mathcal{E}_{u, v}$ ):

$$
\begin{aligned}
& \mathbb{E}_{x}=\mathcal{C}^{2} \mathcal{E}_{u}+\left[\mathcal{S}_{-}^{2}+\mathcal{S}_{+}^{2}-2 \mathcal{S}_{-} \mathcal{S}_{+} \cos \left(q_{+}+q_{-}\right)\right] \mathcal{E}_{v}, \\
& \mathbb{E}_{y}=\mathcal{C}^{2} \mathcal{E}_{v}+\left[\mathcal{S}_{-}^{2}+\mathcal{S}_{+}^{2}-2 \mathcal{S}_{-} \mathcal{S}_{+} \cos \left(q_{+}-q_{-}\right)\right] \mathcal{E}_{u},
\end{aligned}
$$

The following definitions (all $s$ dependent) apply:

$$
\begin{gathered}
\mathcal{C}=\cosh (2 \mathcal{P}), \\
\mathcal{S}_{-}=\frac{\sinh (2 \mathcal{P})}{\mathcal{P}}\left|f_{1001}\right|, \\
\mathcal{S}_{+}=\frac{\sinh (2 \mathcal{P})}{\mathcal{P}}\left|f_{1010}\right|, \\
\mathcal{P}=\sqrt{-\left|f_{1001}\right|^{2}+\left|f_{1010}\right|^{2}}, \\
f_{1001}=\frac{\sum_{w}^{W} J_{w, 1} \sqrt{\beta_{x}^{w} \beta_{y}^{w}} e^{i\left(\Delta \phi_{w, x} \mp \Delta \phi_{w, y}\right)}}{4\left(1-e^{2 \pi i\left(Q_{u} \mp Q_{v}\right)}\right)}, \\
q_{-}=\arg \left\{f_{1001}\right\}, \quad q_{+}=\arg \left\{f_{1010}\right\} .
\end{gathered}
$$

$J_{w}, w=1,2,3 \ldots, W$ are the skew quadrupole integrated strengths present in the ring and originated by quadrupole tilts, sextupole misalignments, insertion devices, and corrector skew quadrupoles already powered. $Q_{u, v}$ are the eigentunes, which are equal to the measured tunes up to the first order in strengths, $Q_{u, v}=Q_{x, y}+O\left(J_{w, 1}^{2}\right)$. $\beta_{r}^{w}$ denotes the Twiss parameter corresponding to the location of the skew quadrupole kick, whereas $\Delta \phi_{w, r}$ is its phase advance with respect to the position where the RDTs $f_{1001}$ and $f_{1010}$ are either measured or computed. Both $\beta_{r}$ and $\phi_{r}$ refer to the ideal, uncoupled lattice. Even though all quantities in Eqs. (6)-(10) are complex numbers, the following relations hold:

$$
1=\mathcal{C}^{2}+\mathcal{S}_{-}^{2}-\mathcal{S}_{+}^{2} ; \quad \mathcal{C}^{2}, \mathcal{S}_{-}^{2}, \mathcal{S}_{+}^{2}, \mathcal{S}_{+} \mathcal{S}_{-} \in \Re,
$$

hence guaranteeing that both apparent emittances of Eqs. (4) and (5) are always real numbers.

\section{B. RMS projected emittances}

Different relations apply for the RMS projected emittances of Eq. (3), namely,

$$
\begin{aligned}
& \epsilon_{x}=\sqrt{\left(\mathcal{C}^{2} \mathcal{E}_{u}+\left[\mathcal{S}_{-}^{2}+\mathcal{S}_{+}^{2}\right] \mathcal{E}_{v}\right)^{2}-\left(2 \mathcal{S}_{+} \mathcal{S}_{-} \mathcal{E}_{v}\right)^{2}}, \\
& \epsilon_{y}=\sqrt{\left(\mathcal{C}^{2} \mathcal{E}_{v}+\left[\mathcal{S}_{-}^{2}+\mathcal{S}_{+}^{2}\right] \mathcal{E}_{u}\right)^{2}-\left(2 \mathcal{S}_{+} \mathcal{S}_{-} \mathcal{E}_{u}\right)^{2}} .
\end{aligned}
$$

In the absence of coupling $f_{1001}=f_{1010}=0, \mathcal{C}=1$ and $\mathcal{S}_{-}=\mathcal{S}_{+}=0$. Only in this case the three emittances coincide, $\quad \epsilon_{r}=\mathcal{E}_{r}=\mathbb{E}_{r}=\sigma_{r}^{2}(s) / \beta_{r}(s)$. The negative terms in Eqs. (13) and (14) shall not be of concern, as they are canceled out after expanding the first parenthesis.

Before analyzing the dependence of the eigenemittances on the RDT, it is worthwhile examining the main differences between the apparent and the projected emittances along the ring in the presence of coupling. As proved in Refs. $[18,19]$, the amplitudes of coupling RDTs remain constant in regions free of coupling sources, while their phases $q_{ \pm}$oscillate with the betatron phases $\left(\phi_{x} \pm \phi_{y}\right)$. When a skew quadrupole kick is met, both the amplitude and phase execute abrupt jumps. This behavior is transmitted to the RMS emittances: As shown in Fig. 1, the apparent emittances oscillate around the ring, because of the terms $\mathcal{S}_{-} \mathcal{S}_{+} \cos \left(q_{+} \pm q_{-}\right)$. This oscillation is of course more important for the vertical apparent emittance, as it is proportional to the larger horizontal eigenemittance $\mathcal{E}_{u}$. The plots refer to the ideal ESRF storage ring with no errors but three localized skew quadrupole kicks. The projected emittance stays constant in the region between the two coupling sources, while the apparent emittance keeps oscillating. When a skew quadrupole kick is met, the projected emittance jumps, while the apparent emittance changes in oscillation amplitude and baseline (because both $\mathcal{S}_{-}$and $\mathcal{S}_{+}$change abruptly). In the bottom

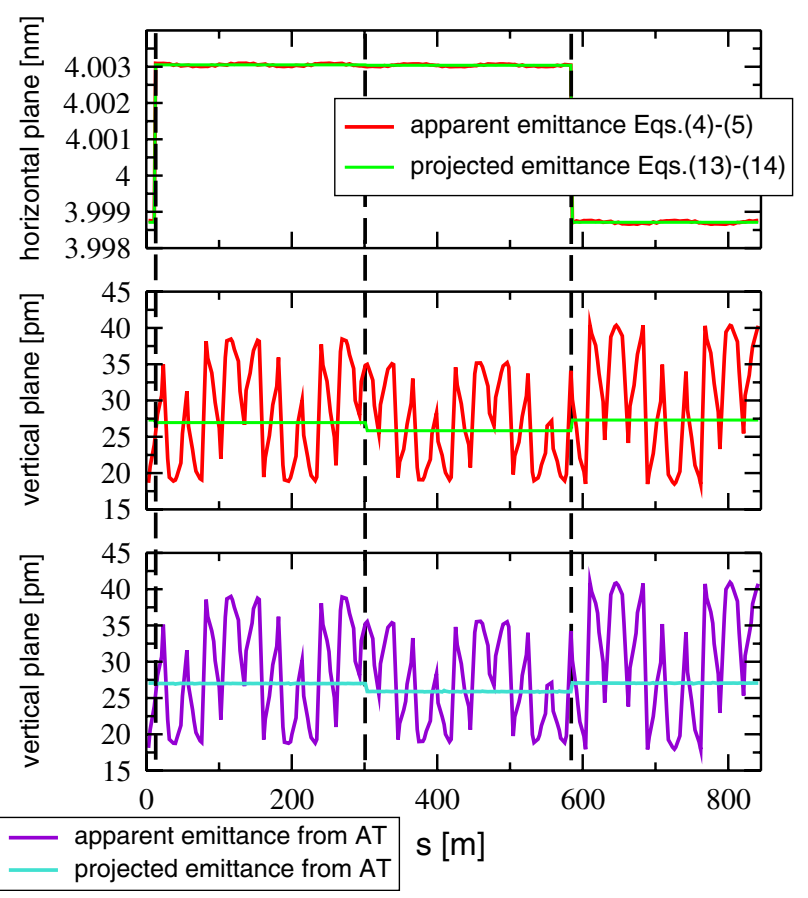

FIG. 1. Example of RMS apparent and projected emittances plotted along the ESRF storage ring. Betatron coupling is driven by three skew quadrupoles whose location is indicated by the vertical dashed lines. In the top and center plots, the horizontal and vertical emittances are evaluated via Eqs. (4), (5), (13), and (14). The bottom plot shows the results for the vertical plane as computed by AT. The corresponding equilibrium emittances are $\mathcal{E}_{u}=4.0 \mathrm{~nm}$ and $\mathcal{E}_{v}=18.4 \mathrm{pm}$. 
plot of Fig. 1, the two vertical emittances as computed by the AT code (implementing the formulas of Ref. [6]) are shown: The agreement between the above formulas and the results of AT is within $0.1 \%$.

Whenever the difference RDT is much larger than the sum term $\left(\left|f_{1010}\right| \ll\left|f_{1001}\right|\right)$ then $\left|\mathcal{S}_{-}\right| \gg\left|\mathcal{S}_{+}\right|$and the two emittances coincide, namely, $\epsilon_{y} \simeq \mathbb{E}_{y} \simeq \mathcal{C}^{2} \mathcal{E}_{v}+$ $\mathcal{S}_{-}^{2} \mathcal{E}_{u}$. This is the typical case of hadron circular accelerators with the two betatron tunes having the same integer part, already treated in Ref. [20].

Another interesting feature of the apparent emittance is that the larger the coupling is (i.e. the product $\mathcal{S}_{-} \mathcal{S}_{+}$), the larger is the oscillation amplitude. This has an operational impact. In fact, the RMS projected vertical emittance is not directly measurable from beam profiles, without a detailed knowledge of coupling sources. Nevertheless its mean value (average over the entire ring)

$$
\overline{\boldsymbol{\epsilon}}_{y}=\frac{1}{C} \oint \epsilon_{y}(s) d s
$$

with $C$ the ring circumference, can be directly estimated from the apparent emittances measured with beam profile monitors, provided that their number and positions are sufficient to cover the full oscillation pattern. Ideally, averaging the measured apparent emittances over a large number of profile monitors $(N \gg 1)$ shall provide a good estimation of $\bar{\epsilon}_{y}$, the oscillating term $\mathcal{S}_{-} \mathcal{S}_{+} \cos \left(q_{+} \pm q_{-}\right)$ averaging to zero. After merging Eqs. (5) and (14) the following approximation can be made:

$$
\begin{aligned}
\bar{\epsilon}_{y} & \simeq\left\langle\mathbb{E}_{y}\right\rangle=\frac{1}{N} \sum_{n=1}^{n=N} \mathbb{E}_{y, n}, \quad \text { with } N \gg 1, \\
\delta \epsilon_{y} & =\sqrt{\frac{1}{N} \sum_{n=1}^{n=N}\left(\mathbb{E}_{y, n}-\left\langle\mathbb{E}_{y}\right\rangle\right)^{2}} \\
& \propto \frac{1}{\sqrt{N}}\left\langle\mathcal{S}_{-} \mathcal{S}_{+} \cos \left(q_{+}-q_{-}\right)\right\rangle \sqrt{\mathcal{E}_{u} \mathcal{E}_{v}},
\end{aligned}
$$

where $\delta \epsilon_{y}$ may be considered as the standard deviation among the measured apparent emittances. On the other hand, if a very limited number of monitors is available ( $N=1$ or 2 , for instance), the averaging of the measured apparent emittance values may underestimate or overestimate the actual mean projected emittance. These two extreme cases are depicted in Fig. 2. There $\mathbb{E}_{y}$ (red) from Eq. (5) and $\epsilon_{y}$ (green) from Eq. (14) are plotted along the ESRF storage ring. The upper plot refers to a well corrected machine with $\mathcal{E}_{v}=3.93 \mathrm{pm} \quad\left(\mathcal{E}_{u}=4.2 \mathrm{~nm}\right)$, whereas larger coupling and vertical dispersion are introduced in the lower plot, with $\mathcal{E}_{v}=9.25 \mathrm{pm}$. As suggested by Eqs. (16) and (17), the ideal average of $\mathbb{E}_{y}$ along the ring approximates well $\bar{\epsilon}_{y}$ : the smaller the coupling, the better the approximation. In order to emphasize the error induced

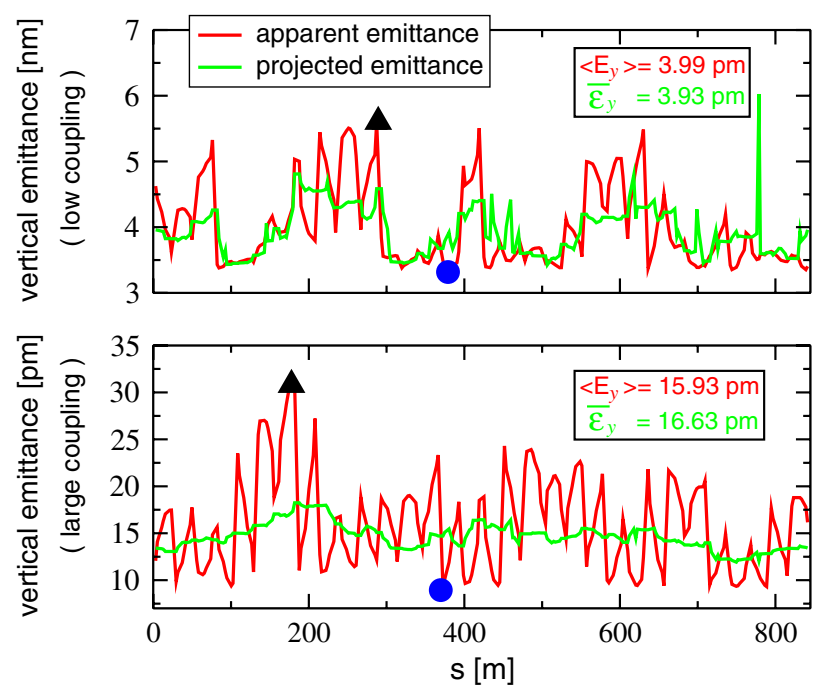

FIG. 2. $\mathbb{E}_{y}$ (red) from Eq. (5) and $\epsilon_{y}$ (green) from Eq. (14) plotted along the ESRF storage ring, for two different amounts of coupling inferred from ORM measurements. The black triangles indicate the positions where $\mathbb{E}_{y}$ would overestimate the average projected emittance $\bar{\epsilon}_{y}$, that on the other hand would be instead underestimated by measurements at the blue circles.

in evaluating the projected emittance by a single measurement of the apparent emittance, a few peculiar observation points have been highlighted. The black triangles indicate the positions where $\mathbb{E}_{y}$ would overestimate the average projected emittance $\bar{\epsilon}_{y}$ (of about $40 \%$ with low coupling, and $100 \%$ with larger coupling). Measurements at the blue circles would instead underestimate $\bar{\epsilon}_{y}(16 \%$ and $50 \%$ in the two cases, respectively).

It is however of general validity the following statement: the larger the coupling is, the larger is the spread among the measured apparent emittances, $\delta \epsilon_{y}$ (provided that monitors are evenly distributed along the ring). $\delta \epsilon_{y}$, rather than the mean projected emittance $\bar{\epsilon}_{y}$, may be used as a qualitative measure of coupling. Imagine measuring the apparent vertical emittance at ten different monitors. A hypothetical first measurement providing $\bar{\epsilon}_{y} \pm \delta \epsilon_{y}=7 \pm$ $2 \mathrm{pm}$ may indicate a well corrected coupling. A later measurement yielding $\bar{\epsilon}_{y} \pm \delta \epsilon_{y}=25 \pm 2 \mathrm{pm}$ should not imply larger coupling, because the spread has not changed (2 pm), whereas coupling would have increased simultaneously both $\bar{\epsilon}_{y}$ and $\delta \epsilon_{y}$, through $\mathcal{S}_{ \pm}$and $\mathcal{E}_{v}$ in Eq. (17). The origin of the larger emittance in the last example should instead be related to other issues, such as vacuum, vertical feedback failures, or instabilities. This is an usual case at the ESRF when an electron beam with uniform filling pattern (992 bunches) is put in operation. Typically, after coupling correction is performed at $20 \mathrm{~mA}$ with 330 bunches only, $\bar{\epsilon}_{y} \pm \delta \epsilon_{y}=6 \pm 1.5 \mathrm{pm}$ is achieved, where the spread $\delta \epsilon_{y}$ is evaluated from Eq. (17) and the apparent emittances measured at 11 monitors. When all 992 bunches 
are stored a the nominal current of $200 \mathrm{~mA} \bar{\epsilon}_{y} \pm \delta \epsilon_{y}=$ $25 \pm 1.5 \mathrm{pm}$, because of instabilities induced by ion trapping. After switching on the vertical bunch-by-bunch feedback, the value usually reduces to $\bar{\epsilon}_{y} \pm \delta \epsilon_{y}=$ $9 \pm 1.5 \mathrm{pm}$. Three different mean projected emittances $(6,20$, and $9 \mathrm{pm})$ are the result of three different sources, whereas $\delta \epsilon_{y}$ remains unchanged, as unchanged is the amount of coupling in the machine. Similarly, when only 4 or 16 bunches are stored (each of 10 and $5.6 \mathrm{~mA}$, respectively), the vertical shaker used for tune measurement is fed with a white noise generator in order to blow up vertically the beam and to augment the Touscheck lifetime. The result is a larger mean apparent vertical emittance (i.e. larger beam size), but almost unchanged spread $\delta \epsilon_{y}$.

\section{Equilibrium emittances}

Both the $s$-dependent apparent and projected emittances depend on the two invariant equilibrium emittances $\mathcal{E}_{u}$ (horizontal mode) and $\mathcal{E}_{v}$ (vertical mode). They are intrinsic properties of the accelerator lattice. The same analytical formulas of Refs. [8,9] are used here. For consistency with Eqs. (4), (5), (13), and (14), they have been translated into the RDT notation in Appendix B. In the absence of coupling, the two read (details may be found in Ref. [8])

$$
\begin{aligned}
& \mathcal{E}_{u}=\frac{1}{2} \frac{\oint\left\{\mathcal{H}_{x}^{2}(s) \mathrm{d}(s)\right\} d s}{\oint\left\{\mathrm{b}_{\mathrm{rf}}(s)-D_{x}(s) \mathrm{b}_{\delta x}(s)\right\} d s}, \\
& \mathcal{E}_{v}=\frac{1}{2} \frac{\oint\left\{\mathcal{H}_{y}^{2}(s) \mathrm{d}(s)\right\} d s}{\oint\left\{\mathrm{b}_{\mathrm{rf}}(s)-D_{y}(s) \mathrm{b}_{\delta y}(s)\right\} d s} .
\end{aligned}
$$

$\mathcal{E}_{v}=0$ if $D_{y}(s) \equiv 0$ anywhere in the ring. The diffusion coefficient reads

$$
\mathrm{d}(s)=\frac{55}{48 \sqrt{3}} \alpha_{0} \frac{\gamma^{5}}{|\rho(s)|^{3}}\left(\frac{\hbar}{m c}\right)^{2},
$$

with $\alpha_{0}$ the fine structure constant, $\gamma$ the relativistic energy factor, $\rho(s)$ the bending radius, $\hbar$ the reduced Planck constant, and $m$ the electron rest mass. $\mathrm{d}(s)$ and hence diffusion are nonzero only inside bending magnets. $\mathcal{H}_{r}$ is the dispersion invariant, defined as

$$
\mathcal{H}_{r}=\gamma_{r} D_{r}^{2}+2 \alpha_{r} D_{r} D_{r}^{\prime}+\beta_{r} D_{r}^{\prime},
$$

with $\gamma_{r}, \beta_{r}$, and $\alpha_{r}$ the Twiss parameters. The transverse damping coefficient generated by rf cavities reads

$$
\mathrm{b}_{\mathrm{rf}}(s)=\frac{1}{c P_{0}} \sum_{i} U_{0, i} \delta\left(s-s_{i, \mathrm{cav}}\right),
$$

where $E_{0}=c P_{0}$ is the reference energy of the particle, $U_{0, i}$ is the energy loss between two rf cavities $\left(\sum_{i} U_{0, i}=U_{0}\right.$ is the net energy loss), and the Dirac function is inserted to localize the damping terms to the corresponding rf cavities. The horizontal damping coefficient due to the bending magnets generating a vertical magnetic field $B_{y}$ reads

$$
\mathrm{b}_{\delta x}(s)=\frac{P_{\gamma}}{2 c E_{0}}\left(\frac{1}{\rho(s)}+\frac{2}{B_{y}(s)} \frac{\partial B_{y}(s)}{\partial x}\right),
$$

where $P_{\gamma}$ is the instantaneous radiated power given by

$$
P_{\gamma}=\frac{e^{2} c^{3} C_{\gamma}}{2 \pi} E_{0}^{2} B_{y}^{2}
$$

and is nonzero in the bending magnets only. $C_{\gamma}=$ $\left(4 \pi r_{e}\right) /\left[3\left(m c^{2}\right)^{3}\right]=8.85 \times 10^{-5} \mathrm{~m} /\left(\mathrm{GeV}^{3}\right)$, with $r_{e}$ the electron classical radius. The first term in Eq. (23) accounts for larger damping experienced by particles passing through the bending magnets are larger $x$, whereas the second term defines the larger radiated power whenever $B_{y}$ contains a quadrupole component. Whenever bending magnets provide vertical focusing too, the vertical damping coefficient shall be included:

$$
\mathrm{b}_{\delta y}(s)=\frac{P_{\gamma}}{c E_{0}} \frac{1}{B_{x}(s)} \frac{\partial B_{x}(s)}{\partial y} .
$$

In Appendix B expressions for the equilibrium emittances in the presence of coupling (and hence of vertical dispersion) using the RDT formalism are derived from Ref. [8], yielding

$\mathcal{E}_{u}=\frac{1}{2} \frac{\oint \mathrm{d}(s)\left\{\mathcal{C}^{2} \mathcal{H}_{x}^{2}(s)+\left[\mathcal{S}_{-}^{2}+\mathcal{S}_{+}^{2}\right] \mathcal{H}_{y}^{2}(s)\right\} d s}{\left.\oint \mathrm{b}_{\mathrm{rf}}(s)-\mathcal{C}^{2} D_{x}(s) \mathrm{b}_{\delta x}(s)-\left[\mathcal{S}_{-}^{2}-\mathcal{S}_{+}^{2}\right] D_{y}(s) \mathrm{b}_{\delta y}(s)\right\} d s}$,

$\mathcal{E}_{v}=\frac{1}{2} \frac{\oint \mathrm{d}(s)\left\{\mathcal{C}^{2} \mathcal{H}_{y}^{2}(s)+\left[\mathcal{S}_{-}^{2}+\mathcal{S}_{+}^{2}\right] \mathcal{H}_{x}^{2}(s)\right\} d s}{\oint\left\{\mathrm{b}_{\mathrm{rf}}(s)-\mathcal{C}^{2} D_{y}(s) \mathrm{b}_{\delta y}(s)-\left[\mathcal{S}_{-}^{2}-\mathcal{S}_{+}^{2}\right] D_{x}(s) \mathrm{b}_{\delta x}(s)\right\} d s}$.

In the above integrands, terms oscillating with $q_{+}$and $q_{-}$have been ignored, as their integrals are usually negligible. $\mathcal{C}, \mathcal{S}_{-}$, and $\mathcal{S}_{+}$are the same of Eqs. (6)-(8) and are $s$ dependent as well as all other quantities in the integrands.

In the same appendix comparisons between the eigenemittances predicted by Eqs. (26) and (27) and by MADX are reported for two cases, one (using the ESRF storage ring lattice) with equal transverse partition numbers, and another one with combined-function magnets and lower horizontal partition number.

\section{From skew quadrupole lattice model to emittances: Application manual}

With all basic formulas introduced in the previous sections it is now possible to define a procedure for the evaluation of the transverse emittances (equilibrium, projected, and apparent) starting from the accelerator model.

(i) Compute the Twiss parameters from the ideal, uncoupled lattice, $\beta_{r}, \alpha_{r}$ and $\gamma_{r}$ ( $r$ stands for either $x$ or $y$ ) from any accelerator optics code, such as MADX, AT, and the like. 
(ii) Include a lattice error model. This may be inferred from beam-based measurements [such as turn-by-turn beam-position-monitor (BPM) data $[19,20]$ or orbit measurements discussed in Sec. III], from measured skew quadrupole magnetic errors, misalignments and tilts, or from their estimation (during design phases). Localized skew quadrupole integrated strengths along the ring, $J_{w, 1}$, are then defined.

(iii) $J_{w, 1}$ are used together with the uncoupled Twiss parameter of step (i) to compute the coupling RDTs $f_{1001}$ and $f_{1010}$ from Eq. (10), and hence to evaluate $\mathcal{C}, \mathcal{S}_{-}$, and $\mathcal{S}_{+}$from Eqs. (6)-(8), and $q_{ \pm}$from Eq. (11).

(iv) Before computing the equilibrium emittances, it is necessary to evaluate the dispersion invariants $\mathcal{H}_{r}$. Notice that vertical dispersion has a double source, one from uncoupled lattice with installation or orbit errors (such as tilted bending magnets, vertical offsets in quadrupoles, and the like), and another from coupling with horizontal dispersion. Even though measuring dispersion in both planes is straightforward, this is not the case for their derivative $D_{r}^{\prime}$, and hence for their invariants. The most realistic lattice model with errors is then necessary in order to evaluate $\mathcal{H}_{r}(s)$ along the ring by means of optics codes capable to handle coupled linear optics.

(v) After computing all damping and diffusion coefficients of Eqs. (20), (22), and (23), the equilibrium emittances $\mathcal{E}_{u, v}$ are obtained from Eqs. (26) and (27).

(vi) From $\mathcal{E}_{u, v}$ and $\mathcal{C}, \mathcal{S}_{-}$and $\mathcal{S}_{+}$the projected emittances along the ring $\epsilon_{r}(s)$ are deduced via Eqs. (13) and (14).

(vii) The apparent emittances $\mathbb{E}_{r}(s)$ are eventually determined from Eqs. (4) and (5). The RMS beam sizes $\left\langle x^{2}\right\rangle$ and $\left\langle y^{2}\right\rangle$ may be eventually inferred after inverting Eq. (2) and may be compared with measurements.

An alternative approach is the derivation of all different emittances from the error model [i.e. the RDT functions of step (iii)], from the measured dispersion $D_{r}$, and from the measured RMS beam sizes $\left\langle x^{2}\right\rangle,\left\langle y^{2}\right\rangle$. Indeed, after removing the dispersive terms, $\mathbb{E}_{r}(s)$ may be evaluated from Eq. (2). Equations (4) and (5) form a linear system with all quantities known, save the equilibrium emittances, $\mathcal{E}_{u, v}$, that may be hence inferred after inverting the system. Equations (13) and (14) may eventually be used to derive the projected emittances. This approach has the advantage that only the dispersion functions $D_{r}$ (straightforward to measure) are necessary, without need of evaluating the dispersion invariants $\mathcal{H}_{r}$.

\section{MODELING COUPLING VIA ORBIT RESPONSE MATRIX MEASUREMENTS IN THE ESRF STORAGE RING}

In Refs. [17-20] a method to use turn-by-turn beamposition-monitor (TBT-BPM) data to measure RDTs and correct coupling has been already discussed, both theoretically and experimentally. This approach has the advantage of being faster than traditional methods, such as the minimization of $\Delta Q_{\min }$ [21]. The success of BPM measurements is bound to (i) the possibility of acquiring a large number of TBT data with small or no decoherence (typically 512 turns), in order to enhance their spectral resolution, and (ii) to an easy (as well as rapid) BPM electronic switch from orbit mode to TBT mode. The first condition is hardly met in electron storage rings like the one of the ESRF. Operational settings require indeed strong chromatic sextupoles and nonzero detuning with amplitude in order to guarantee sufficient longitudinal acceptance. Detuning being responsible for decoherence, it is already challenging to achieve 100 turns with exploitable TBTBPM data. Moreover, the ESRF storage ring, like other European third-generation light sources, is equipped with the LIBERA BPM system [22], whose TBT mode was not fully operational until the second half of 2010.

This led to the development of a lattice error model obtained from ORM measurements similar to the one of Ref. [23], which in the case of the ESRF storage ring requires about 20 minutes. The accelerator consists of 32 focusing cells, each comprising eight main quadrupoles. In order to provide straight sections for insertion devices of both low and high $\beta_{x}$, each downstream cell is the mirrored copy of the one upstream, hence leaving a 16 -fold periodicity. More details on the lattice and its recent upgrade can be found in Ref. [24]. Each cell is equipped with seven dual-plane BPMs (224 over the entire ring). The ORM is measured after powering 32 horizontal and 32 vertical steerers and recording the orbit distortion at all 224 BPMs. The resulting ORM is then a $(32 \cdot 2) \times(224 \cdot 2)$ matrix:

$$
\mathrm{ORM}=\left(\begin{array}{ll}
\mathbf{O}_{\mathbf{x x}} & \mathbf{O}_{\mathbf{x y}} \\
\mathbf{O}_{\mathbf{y x}} & \mathbf{O}_{\mathbf{y y}}
\end{array}\right) .
$$

Normal quadrupole errors in the $8 \cdot 32=256$ main quadrupoles are inferred from the diagonal blocks $\mathbf{O}_{\mathbf{x x}}$ and $\mathbf{O}_{\mathbf{y y}}$ via SVD. The off-diagonal blocks $\mathbf{O}_{\mathbf{x y}}$ and $\mathbf{O}_{\mathbf{y x}}$ result from coupling generated by tilts in the main quadrupoles and sextupoles, and by vertical off-axis orbit and/or misalignments in sextupoles. In each cell, however, the three chromatic sextupoles with the strongest fields are placed next to quadrupoles. In practice, then, only quadrupole tilts are fit via SVD in order to best match the offdiagonal blocks, assuming that those next to the chromatic sextupoles play the role of effective tilts accounting for both magnets.

The difference between the measured vertical dispersion and the one obtained including coupling errors is eventually attributed to tilts in the main bending magnets.

Normal quadrupole errors $\Delta K_{1}$, quadrupole tilts $\theta$, and bending magnet rotations $\omega$ provide the lattice error model that best matches the ORM and vertical dispersion. As a check of the model effectiveness, this is included in the AT optics model and the predicted vertical apparent emittances are compared with the ones measured at different 


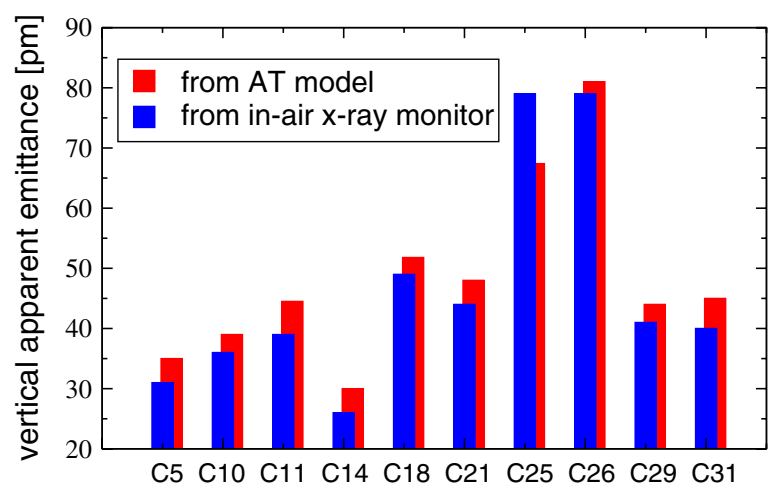

FIG. 3. Example of comparison between the apparent emittances $\mathbb{E}_{y}$ (before coupling correction) measured at ten available inair x-ray detectors (blue) and the predictions of AT (red) after creating the lattice error model from the ORM measurement of January 16, 2010.

devices: two x-ray pinhole cameras (D09, D25), plus 11 in-air x-ray detectors $(\mathrm{C} 03, \mathrm{C} 05, \ldots, \mathrm{C} 31)$ placed along the ring. Details on these diagnostic tools can be found in Ref. [25]. A typical result before coupling correction is shown in Fig. 3.

The distribution of skew quadrupole strengths along the machine is derived as follows:

$$
J_{w, 1}=-\left[K_{w, 1}+\Delta K_{w, 1}\right] \sin \left(2 \theta_{w}\right),
$$

where the index $w=1,2, \ldots, 256$ corresponds to the position of the 256 main quadrupoles, where the skew quadrupole integrated strengths are inserted. Following the procedure described in Sec. II D, the projected emittances may be evaluated together with all other optics parameters.

\section{SCHEME FOR COUPLING CORRECTION AND VERTICAL EMITTANCE MINIMIZATION}

The ESRF storage ring is equipped with 32 independent corrector normal quadrupoles to compensate focusing errors induced by $\Delta K_{w, 1}$. Coupling (and vertical dispersion) correction is performed by means of 32 independent skew quadrupoles, distributed rather uniformly around the ring.

Until the end of 2009 coupling correction was performed by minimizing along the ring either the vertical eigenemittance or the apparent one (as computed by AT) via the Matlab function FMINSEARCH. The dependence of the vertical emittances on the corrector strengths being quadratic, this resulted in a nonlinear multidimensional minimization over 32 parameters. The main drawbacks of this approach are CPU time (about $10 \mathrm{~min}$ for 500 iterations) and the risk of limited improvements whenever a local minimum (not necessarily the lowest) is found.

Equations (5) and (14) suggest an intuitive consideration: the lower the RDTs (i.e. the coupling), the lower the vertical emittance, as the contribution from the large horizontal equilibrium emittance $\mathcal{E}_{u}$ is minimized. A setting for the 32 skew quadrupole correctors may be then found to minimize as uniformly as possible both coupling RDTs along the ring. The system to invert via SVD reads

$$
\left(\begin{array}{l}
\vec{f}_{1001} \\
\vec{f}_{1010}
\end{array}\right)_{\text {meas }}=-\mathbf{M} \vec{J}_{c},
$$

where $\vec{J}_{c}=\left(J_{1}^{(1)}, \ldots, J_{1}^{(c)}, \ldots, J_{1}^{(32)}\right)$ are the integrated strengths of the 32 corrector skew quadrupoles to be determined, $\vec{f}^{T}=\left(f^{(1)}, \ldots, f^{(w)}, \ldots, f^{(224)}\right)$ is a 224 (complex) vector containing the measured or computed RDT at all BPMs, and $\mathbf{M}$ is the (complex) RDT $(224 \cdot 2) \times 32$ response matrix, whose generic element according to Eq. (10) reads

$$
\begin{aligned}
& m_{w, c}=\frac{\sqrt{\beta_{x}^{(c)} \beta_{y}^{(c)}} e^{i\left(\Delta \phi_{w, x}^{(c)}-\Delta \phi_{w, y}^{(c)}\right)}}{4\left(1-e^{2 \pi i\left(Q_{u}-Q_{v}\right)}\right)} \quad \text { for } w \leq 224, \\
& m_{w, c}=\frac{\sqrt{\beta_{x}^{(c)} \beta_{y}^{(c)}} e^{i\left(\Delta \phi_{w, x}^{(c)}+\Delta \phi_{w, y}^{(c)}\right)}}{4\left(1-e^{2 \pi i\left(Q_{u}+Q_{v}\right)}\right)} \quad \text { for } w>224,
\end{aligned}
$$

where $\beta^{(c)}$ is the beta function at the location of the skew corrector $c$, and $\Delta \phi_{w}^{(c)}$ is the phase advance between the same corrector and the BPM $w$. By inverting via SVD the linear system of Eq. (30) the strengths for the corrector magnets that best reduce the coupling RDTs are derived.

By itself coupling correction implies that $\mathcal{C}^{2} \simeq 1, \mathcal{S}_{ \pm}^{2} \simeq 0$, and hence that $\epsilon_{y} \simeq \mathbb{E}_{y} \simeq \mathcal{E}_{v}$. This, however, is not sufficient, as the eigenemittance $\mathcal{E}_{v}$ is minimized only after a further correction of vertical dispersion, i.e., after minimizing $\mathcal{H}_{y}$, see Eq. (27). Skew quadrupoles may still be used to this end. Indeed, Eq. (30) may be generalized as follows:

$$
\left(\begin{array}{c}
a_{1} \vec{f}_{1001} \\
a_{1} \vec{f}_{1010} \\
a_{2} \vec{D}_{y}
\end{array}\right)_{\text {meas }}=-\mathbf{M} \vec{J}_{c},
$$

where $\mathbf{M}$ is now a $(224 \cdot 2+224) \times 32$ matrix. The generic element of the additional $224 \times 32$ block reads

$$
m_{w, c}=\frac{\Delta D_{y}^{(w)}}{\Delta J_{1}^{(c)}},
$$

where $\Delta D_{y}^{(w)}$ is the vertical dispersion distortion at the BPM number $w$ induced by the skew corrector strength $\Delta J_{1}^{(c)}$. These terms need to be computed by means of optics codes, as they depend on the error lattice model. The weights $a_{2}=1-a_{1}$ are introduced in order to determine the best compromise between correction of dispersion and deterioration of coupling. Their determination is empirical and in the case of the ESRF storage ring the best correction is found for $a_{2}=0.7$. Note that the system of Eq. (33) is analogous to the one already proposed and successfully implemented in Ref. [14], with the difference of having the RDTs instead of the vertical orbit distortion to be 
minimized. The system being linear in the corrector strengths, it is rapid (a few CPU seconds only) and does not require any iteration, as it determines directly the lowest minimum for the RDTs along the ring. Attempts to perform the nonlinear minimization on top of the RDT correction did not provide significant improvements.

A coupling correction would be of limited help if focusing errors are not previously compensated. In analogy with their skew peers, normal quadrupole errors excite two RDTs, $f_{2000}$ and $f_{0020}$, in the horizontal and vertical planes, respectively [26]. They are so defined

$$
\begin{aligned}
& f_{2000}=\frac{\sum_{w}^{W} \Delta K_{w, 1} \beta_{x}^{w} e^{2 i \Delta \phi_{w, x}}}{8\left(1-e^{4 \pi i Q_{x}}\right)}, \\
& f_{0020}=\frac{\sum_{w}^{W} \Delta K_{w, 1} \beta_{y}^{w} e^{2 i \Delta \phi_{w, y}}}{8\left(1-e^{4 \pi i Q_{y}}\right)},
\end{aligned}
$$

where $\Delta K_{w, 1}$ are the focusing errors inferred from the ORM and the strengths of the corrector quadrupoles already powered. Focusing errors may then be corrected by solving via SVD the following system:

$$
\left(\begin{array}{l}
\vec{f}_{2000} \\
\vec{f}_{0020}
\end{array}\right)_{\text {meas }}=-\mathbf{N} \vec{K}_{c} \text {, }
$$

where $\vec{K}_{c}=\left(K_{1}^{(1)}, \ldots, K_{1}^{(c)}, \ldots, K_{1}^{(32)}\right)$ are the integrated strengths of the 32 corrector normal quadrupoles to be determined, and $\mathbf{N}$ is the (complex) RDT $(224 \cdot 2) \times 32$ response matrix, whose generic element according to Eqs. (35) and (36) reads

$$
\begin{aligned}
& m_{w, c}=\frac{\beta_{x}^{(c)} e^{i\left(\Delta \phi_{w, x}^{(c)}\right)}}{8\left(1-e^{4 \pi i Q_{x}}\right)} \quad \text { for } w \leq 224 \\
& m_{w, c}=\frac{\beta_{y}^{(c)} e^{i\left(\Delta \phi_{w, y}^{(c)}\right)}}{8\left(1-e^{4 \pi i Q_{y}}\right)} \quad \text { for } w>224
\end{aligned}
$$

\section{RESULTS FROM THE 2010 CAMPAIGN AT THE ESRF ELECTRON STORAGE RING}

The correction scheme of Sec. IV for both focusing errors and coupling was tested for the first time during the machine startup of January 2010 after the winter shutdown. First, an ORM measurement with the latest corrector setting of 2009 was launched in order to verify the error model, by comparing the vertical apparent emittances from the AT model and the ones measured at the in-air x-ray monitors. Results have been already shown in Fig. 3. All measurements and correction discussed in this sections were carried out with open ID gaps, hence referring to the bare machine.

In order to test the effectiveness of the new proposed scheme, all correctors (both 32 normal and 32 skew quadrupoles) were switched off. Despite the large errors (beta beating of about $50 \%$ and vertical apparent emittances of the order of hundreds of pm) stable beam could be stored. The vertical apparent emittance measured along the ring before the first correction is shown in the upper left plot of Fig. 4, providing an average emittance, as defined in Eqs. (16) and (17), $\left(\bar{\epsilon}_{y} \pm \delta \epsilon_{y}\right)=237 \pm 122 \mathrm{pm}$. Error bars on individual monitors are derived from the inferred beta beating. After a first focusing correction, beta beating went down to about $8 \%$, and $\left(\bar{\epsilon}_{y} \pm \delta \epsilon_{y}\right)=23.6 \pm 6.3 \mathrm{pm}$, see center left plot of Fig. 4. A second ORM measurement and correction was then applied providing a further reduction of coupling, yielding $\left(\bar{\epsilon}_{y} \pm \delta \epsilon_{y}\right)=11.5 \pm 4.3 \mathrm{pm}$, as shown in the bottom left plot of Fig. 4, while no further improvement was observed in the beta beating. The amplitudes of the coupling RDTs corresponding to the three cases are displayed in the right graphs.

A further ORM measurement and correction did not reduce significantly the latest results. On the contrary, a slow vertical emittance increase was observed without any further change in the corrector magnets. This was suspected to be related to a slow vacuum instability due to the limited time of conditioning (three days after shutdown). The absence of such a slow increase after a few weeks of operations supported this conjecture. In Table I the main observables measured during the experiment of January 16th are summarized.

In further optimization tests it was possible to bring all vertical apparent emittances measured at the in-air x-ray monitors well below $10 \mathrm{pm}$, whereas the values reported by the two pinhole cameras would stay constantly well above. It was suspected that at very low vertical emittance, the installed vertical pinhole of $25 \mu \mathrm{m}$ might define a lower limit to the measurable beam size. To confirm this conjecture, a test was carried out on the D25 pinhole camera in February 10th. With the $25 \mu \mathrm{m}$ pinhole a stable value of $\mathbb{E}_{y}(\mathrm{D} 25)=17 \mathrm{pm}$ was reported. After switching to a $10 \mu \mathrm{m}$ aperture, the read value dropped to $\mathbb{E}_{y}(\mathrm{D} 25)=$ $9.5 \mathrm{pm}$. With this confirmation it was however decided to remove both cameras in the computation of the average emittance for very low values around $5 \mathrm{pm}$, in order to avoid further systematic errors induced by the finite pinhole size.

The lowest vertical emittance ever measured at the ESRF was obtained during the machine studies of July 22, 2010, after minimizing coupling RDTs and vertical dispersion, again at open ID gaps. Results are shown in Fig. 5 (note that the C05 monitor of January 16th was out of order and replaced by C03). In the plot it is still possible to observe how the apparent emittance measured at the two pinhole cameras equipped with a $10 \mu \mathrm{m}$ aperture are still about a factor 2 larger than the values reported by the $\mathrm{x}$-ray monitors. The monitor in cell 25 reported $\mathbb{E}_{y}(\mathrm{C} 25)=$ $4.6 \pm 0.5 \mathrm{pm}$, while the neighbor camera provided $\mathbb{E}_{y}(\mathrm{D} 25)=9.5 \pm 0.5 \mathrm{pm}$. By computing the mean vertical emittance from the available ten $\mathrm{x}$-ray monitors the following value was derived: 

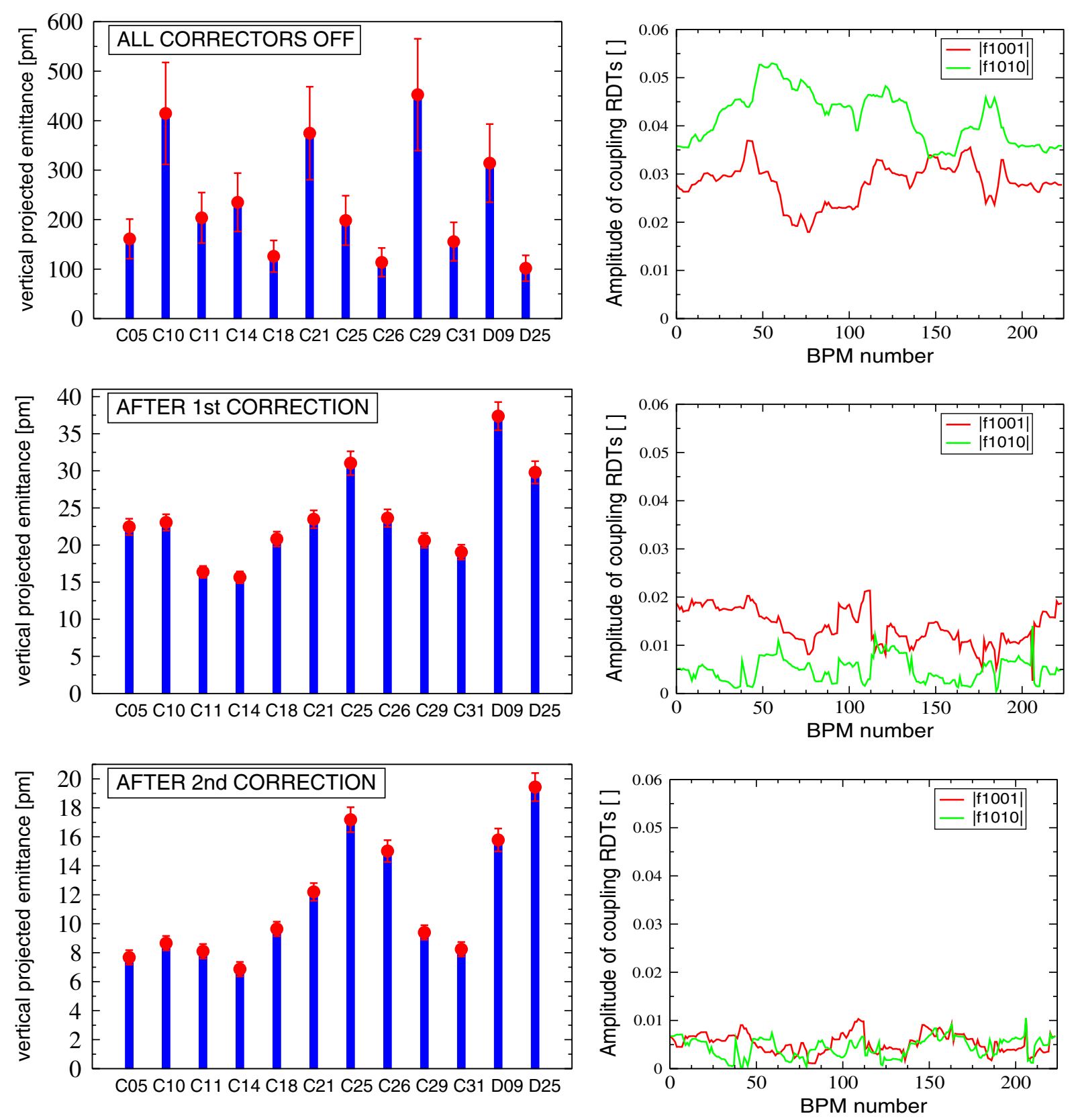

FIG. 4. Left column: Vertical apparent emittances $\mathbb{E}_{y}$ measured along the ESRF storage ring with all correctors off (upper plot), after first correction with minimization of coupling RDTs and vertical dispersion (center plot), and after a second measurement and correction (bottom plot). The names in the abscissa refer to the used monitors: ten (out of 11) in-air x-ray monitors (from C05 to C31) and two pinhole cameras (D09 and D25). Right column: Amplitude of the corresponding coupling RDTs.

TABLE I. Summary table of the first correction with RDTs of January 16, 2010. The corresponding horizontal emittance is $\epsilon_{x} \simeq \mathbb{E}_{x} \simeq \mathcal{E}_{u} \simeq 4 \mathrm{~nm}$. Beta beat refers to the peak value.

\begin{tabular}{lcc}
\hline \hline Condition & $\bar{\epsilon}_{y} \pm \delta \epsilon_{y}[\mathrm{pm}]$ & $\beta$ beat [\%] \\
\hline With 2009 correction & $46 \pm 18$ & 5 \\
All correctors OFF & $237 \pm 122$ & 50 \\
After 1st correction & $23.6 \pm 6.3$ & 8 \\
After 2nd correction & $11.5 \pm 4.3$ & 5 \\
\hline \hline
\end{tabular}

$$
\bar{\epsilon}_{y} \pm \delta \epsilon_{y}=(4.4 \pm 0.7) \mathrm{pm}
$$

Being the horizontal emittance measured at both pinhole cameras $\epsilon_{x}=(4.2 \pm 0.2) \mathrm{nm}$, this corresponds to an emittance ratio of about $0.1 \%$.

It is worthwhile noticing that in general at the ESRF storage ring $\left|f_{1010}\right| \approx\left|f_{1001}\right|$ (see right column of Fig. 4), i.e., that it is not possible to neglect the contribution of the coupling sum resonance. This is a general feature of this accelerator, as of any machine with tunes having a large 


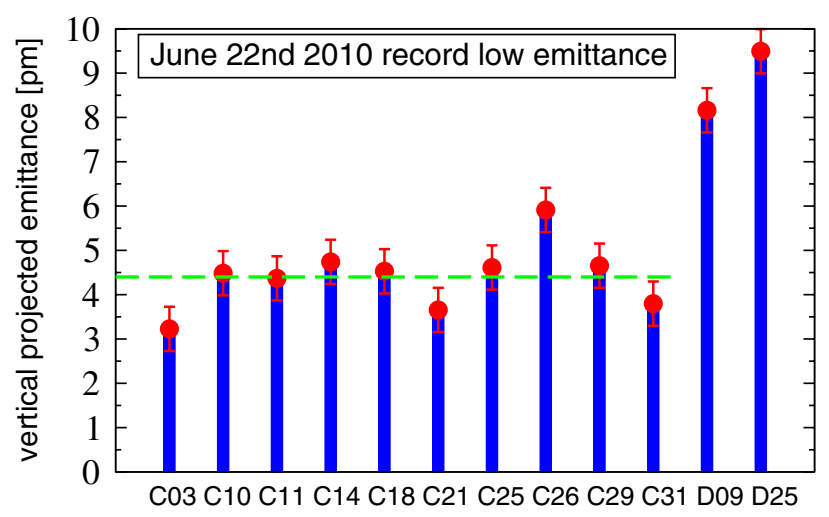

FIG. 5. Record low vertical apparent emittances $\mathbb{E}_{y}$ measured along the ESRF storage ring on July 22, 2010 at ID gaps open. The horizontal dashed line denotes the mean values between the ten (out of 11) available x-ray monitors. The D09 and D25 were taken out of the average, see main text.

difference between the integer parts of the tunes $\left(Q_{x}=\right.$ 36.44 and $Q_{y}=13.39$ in the case of the ESRF). Even in the best case of Fig. 5 the coupling resonance strengths, as defined in Ref. [21], are $\left|C_{+}\right|=3 \times 10^{-3}$ and $\left|C_{-}\right|=$ $0.9 \times 10^{-3}$. By using the same procedure of Ref. [3] to evaluate the vertical emittance from the $\epsilon_{x}$ and $\left|C_{-}\right|$only, the result would be a factor 2 lower than the real one.

\section{PRESERVING LOW VERTICAL EMITTANCE AGAINST ID GAP MOVEMENTS}

As already mentioned in the Introduction, coupling correction at open ID gaps may not last during beam delivery, because of the continuous changes of the vertical apertures. The residual magnetic imperfections in some IDs may
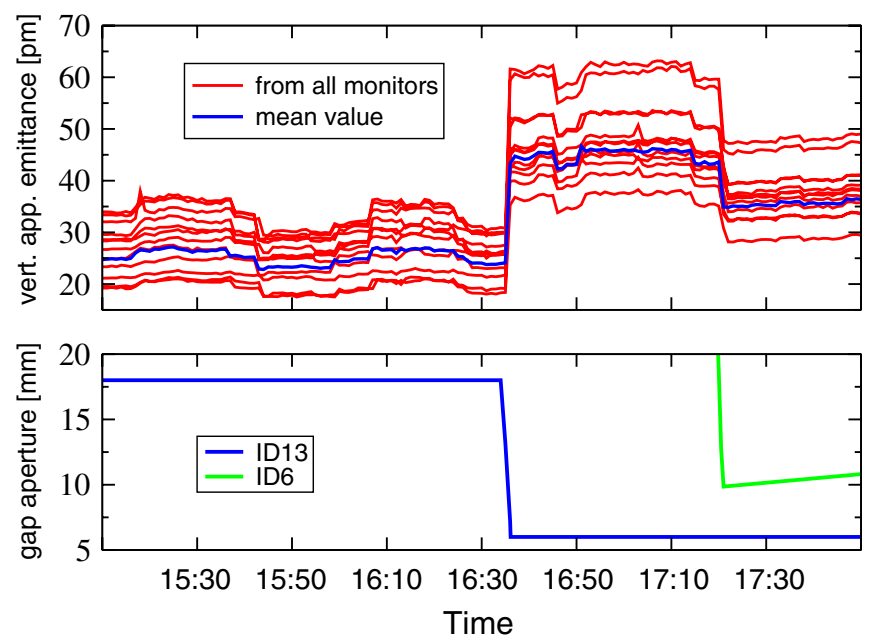

FIG. 6. Example of vertical apparent emittance abrupt jumps (top) following two ID gap movements (bottom). In the upper graph the emittance is measured at 12 monitors (red lines) and the corresponding mean value $\bar{\epsilon}_{y}$ is depicted by the blue line. The corresponding apertures of ID6 and ID13 are reported in the lower plot. include gap-dependent skew quadrupoles terms. This concerns mainly high-field wigglers installed more than ten years ago or small-gap devices such as in-vacuum undulators. An example of the impact of ID gap movements on the apparent vertical emittance recorded at 12 monitors on January 20th during normal beam delivery is shown in Fig. 6. The sudden closure of the ID13 gap from 18 to $6 \mathrm{~mm}$ augments coupling and both $\bar{\epsilon}_{y}$ and $\delta \epsilon_{y}$ increase of about a factor two. The further closure of ID6 about $1 \mathrm{~h}$ later compensates partly the impact of ID13, by reducing both $\bar{\epsilon}_{y}$ and $\delta \epsilon_{y}$ of about $20 \%$.

For the time being, ORM may not be measured during beam delivery. Hence, any automatic correction should be based on the knowledge of the amount of coupling introduced by IDs against the values of their gaps. Correction look-up tables may then be used to compensate for them. Alternatively, the strengths of corrector skew quadrupoles may be dynamically trimmed to minimize $\bar{\epsilon}_{y}$. The first (feed-forward) approach is discussed hereafter, while the practical implementation of the second (feedback) is presented in the second part of this section.

In the ESRF storage rings dual-plane iron-free corrector steerers are installed at both ends of each straight section (see Fig. 7). They are however used in nine regions only, where IDs induce important gap-dependent integrated dipole errors. In this case, dipole correctors are controlled in a feed-forward process using look-up tables created from beam-based measurements. The structure of these correctors is such that they can be configured as skew quadrupole correctors by modifying the electrical wiring between the coils. The integrated skew quadrupole gradient generated in this way is $3.5 \mathrm{mT} / \mathrm{A}$ (from magnetic measurements). Thanks to the excellent coupling correction of the bare machine, any additional coupling induced by an individual ID may be easily quantified and corrected. By repeating the same procedure separately with other IDs, low vertical emittance may be preserved even during beam delivery, betatron coupling being linear.

It is worthwhile mentioning that in principle four skew correctors would be needed in order to compensate the two (complex) RDTs, as two are necessary to compensate each excited resonance. Nevertheless, the vertical phase advance between the two ID ends is usually rather small, hence ensuring that by correcting to contribution to either resonance, the other is automatically compensated.

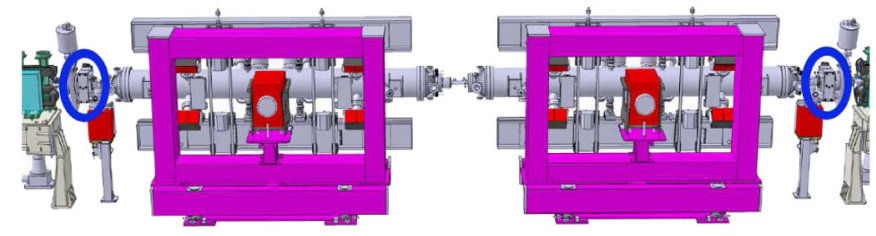

FIG. 7. Drawings of an ESRF straight section housing two undulators. Corrector steerers (blue ellipse) are placed at both ends. 
A proof-of-principle test was carried out on May 5, 2010. The in-vacuum undulator ID6 was chosen as it was known to be one of the most important coupling sources at low gap values. The influence of this ID gap movement on the vertical emittance is reported in Table II and plotted in Fig. 8: At its minimum aperture value, the vertical mean emittance $\bar{\epsilon}_{y}$ and spread $\delta \epsilon_{y}$ were both augmented of about $50 \%$. At each step reported in the table, the setting of the two skew correctors that would bring back the vertical emittance to its initial value was empirically determined and stored in a look-up table. Intermediate values are determined via linear interpolation of the two neighbor measured points. In the most right column of Table II, as well as in Fig. 8, it is shown how effective such a scheme may be. After the successful test, the correction was left in operation during beam delivery and a program for its extension to other IDs has been launched.

A coupling feedback loop recently tested and put in operation consists in dynamically adjusting the 32 skew quadrupole correctors to preserve the lowest average vertical emittance $\bar{\epsilon}_{y}$. Coupling may be described by two complex numbers (vectors) $C_{+}$and $C_{-}$[21], for the sum and difference resonances, respectively, defined as follows:

$$
\begin{aligned}
C_{ \pm} & =\sum_{w}^{W} J_{w, 1} \sqrt{\beta_{x}^{w} \beta_{y}^{w}} e^{i \Phi_{w, \pm},} \\
\Phi_{w, \pm} & =\phi_{w, x} \pm \phi_{w, y}-\left(Q_{x} \pm Q_{y}-N_{ \pm}\right) 2 \pi s_{w} / C,
\end{aligned}
$$

where $\beta$ and $\phi$ are the Twiss parameters, $J_{w, 1}$ are the integrated skew quadrupole strengths (in units of $\mathrm{m}^{-1}$ ), $s_{w}$ denotes the curvilinear abscissa around the ring of the coupling source, $Q_{x, y}$ are the tunes, and $N_{ \pm}$are integer numbers. In the case of the ESRF storage ring $Q_{x}=36.44$, $Q_{y}=13.39, N_{+}=50$, and $N_{-}=23$. The two vectors may be decomposed in

$$
C_{ \pm}=C_{ \pm, \mathrm{b} . \mathrm{m} .}+C_{ \pm, \mathrm{static}}{ }^{\mathrm{corr}}+C_{ \pm, \mathrm{IDs}}(t)+C_{ \pm, \mathrm{tr}}{ }^{\mathrm{corr}}(t) .
$$

TABLE II. Mean vertical emittance $\bar{\epsilon}_{y}$ and spread $\delta \epsilon_{y}$ measured against the vertical aperture of the ID6 in-vacuum undulator. The first column refers to the existing uncorrected setting, while the second was obtained after implementing correction look-up tables.

\begin{tabular}{lcc}
\hline \hline $\begin{array}{l}\text { ID6 undulator } \\
\text { gap [mm] }\end{array}$ & $\begin{array}{c}\bar{\epsilon}_{y} \pm \delta \epsilon_{y}[\mathrm{pm}] \text { without } \\
\text { correction }\end{array}$ & $\begin{array}{c}\bar{\epsilon}_{y} \pm \delta \boldsymbol{\epsilon}_{y} \text { [pm] with } \\
\text { look-up table }\end{array}$ \\
\hline 30 & $5.1 \pm 1.1$ & $4.9 \pm 1.1$ \\
15 & $5.1 \pm 1.1$ & $4.9 \pm 1.1$ \\
10 & $5.6 \pm 1.2$ & $4.8 \pm 1.1$ \\
8 & $6.0 \pm 1.2$ & $4.8 \pm 1.1$ \\
7 & $6.3 \pm 1.3$ & $4.8 \pm 1.1$ \\
6.5 & $6.6 \pm 1.4$ & $4.9 \pm 1.1$ \\
6 & $7.7 \pm 1.6$ & $4.9 \pm 1.1$ \\
\hline \hline
\end{tabular}

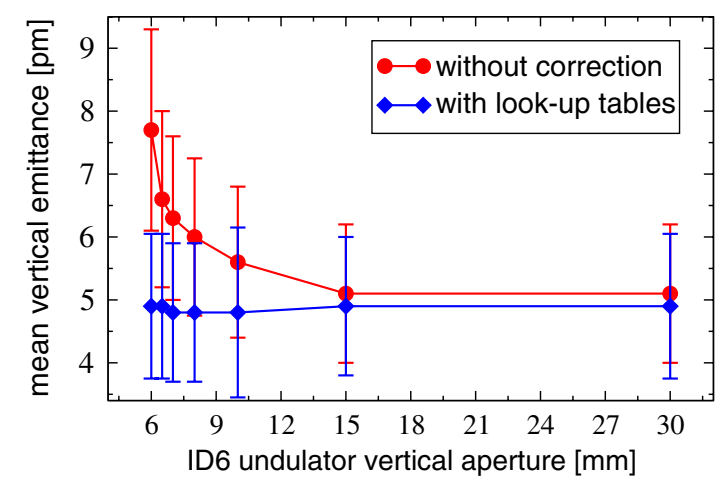

FIG. 8. Mean vertical emittance $\bar{\epsilon}_{y}$ measured against the vertical aperture of the ID6 in-vacuum undulator without any correction (red circles) and with automatic coupling compensation (blue diamonds). Error bars corresponds to the spread $\delta \epsilon_{y}$ of Table II.

$C_{ \pm, \text {b.m. }}$ represents the (static) coupling generated by the bare machine and ID at fixed gap apertures and is corrected via RDT and vertical dispersion minimization by $C_{ \pm, \text {static }}{ }^{\text {corr }}$, as discussed in Sec. IV. Coupling variations induced by ID gap movements induce an additional timedependent $C_{ \pm, \mathrm{IDs}}(t)$. On top of the static correction, the 32 corrector skew quadrupoles may be trimmed to introduce a time-dependent compensation, $C_{ \pm, t r}{ }^{\operatorname{corr}}(t)$ that cancels $C_{ \pm, \mathrm{IDs}}(t)$. The correctors' strengths would then be composed by

$$
J_{w, 1}{ }^{\text {corr }}=J_{w, 1, \text { static }}{ }^{\text {corr }}+J_{w, 1, \text { tr }}{ }^{\operatorname{corr}}(t), \quad w=1, \ldots, 32 .
$$

The time-dependent correction may be then written as

$C_{ \pm, \mathrm{tr}}{ }^{\mathrm{corr}}(t)=\sum_{w=1}^{32} J_{w, 1, \mathrm{tr}}{ }^{\operatorname{corr}}(t) \sqrt{\beta_{x}^{w} \beta_{y}^{w}} e^{i \Phi_{w, \pm}}=A_{ \pm}(t) e^{i \Theta_{ \pm}(t)}$

The four parameters $A_{ \pm}$and $\Theta_{ \pm}$may be varied via a software application (depicted in Fig. 9). The figure of merit for the definition of the best setting is the mean value of the vertical apparent emittances measured at the 11 inair x-ray monitors, $\bar{\epsilon}_{y}$, which is automatically evaluated at the frequency of $1 \mathrm{~Hz}$. Until November 2010, operators would trim $A_{ \pm}$and $\Theta_{ \pm}$by trials and errors. Recently, a coupling feedback loop was installed to periodically vary $A_{-}$and $\Theta_{-}$to minimize $\bar{\epsilon}_{y}$ (the further correction of the sum resonance was found to be of minimal benefit). Figure 10 shows a comparison between the vertical emittance evolution during one week of beam delivery with and without the coupling feedback. In the later case, uncompensated ID gap movements during the first day caused $\bar{\epsilon}_{y}$ to reach $30 \mathrm{pm}$ (from the initial $6 \mathrm{pm}$ ). Low emittance is retrieved only after a manual regulation of $A_{-}$and $\Theta_{-}$. When the automatic loop was activated a few weeks later, $\bar{\epsilon}_{y}$ remained stable between 6 and $7 \mathrm{pm}$. 

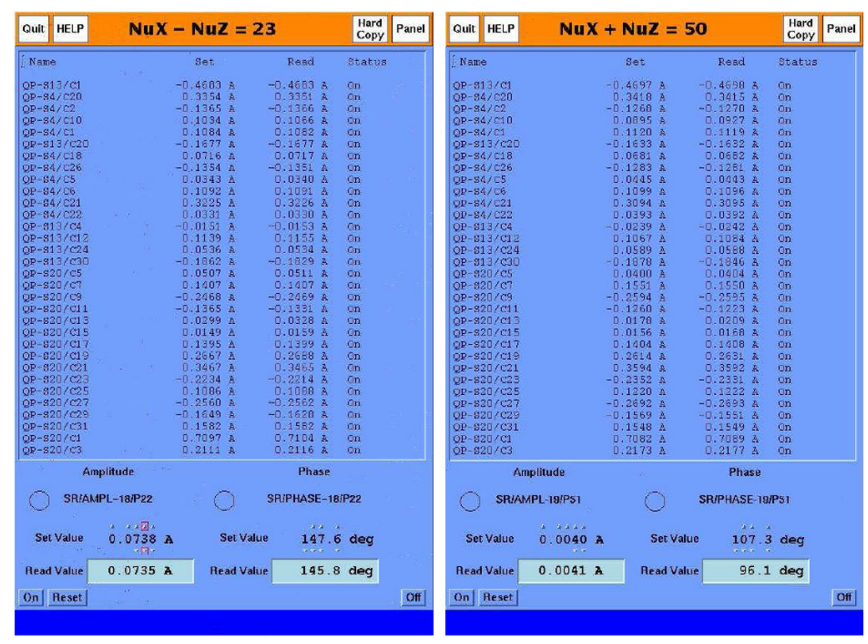

FIG. 9. Snapshot of the software application driving the trim values for the 32 skew quadrupole correctors. Amplitude $A$ (already normalized in terms of magnet current) and phase $\Theta$ for both resonances may be varied either by hand or by a coupling feedback software loop.

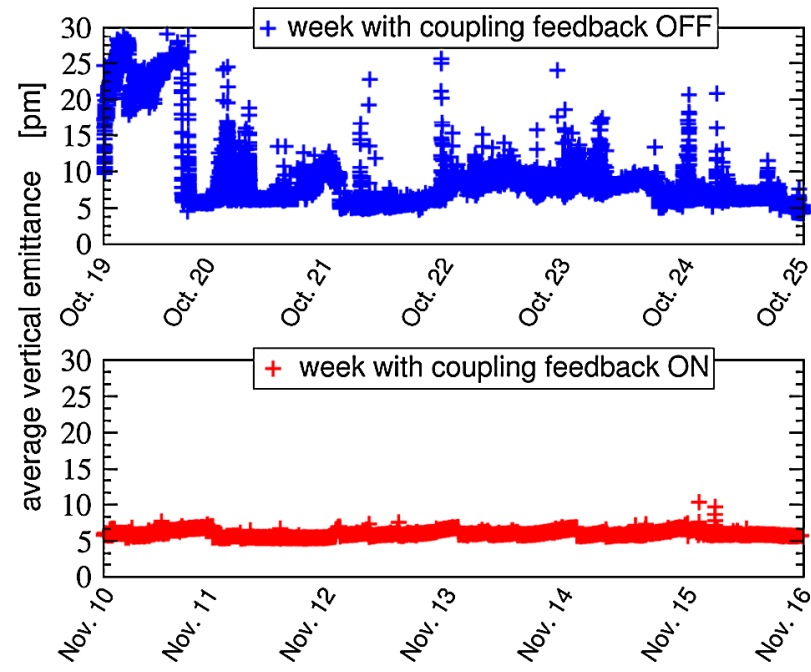

FIG. 10. Comparison between the mean vertical apparent emittance $\bar{\epsilon}_{y}$ measured during beam delivery without (top) and with (bottom) the coupling feedback. Data acquired during injections are not displayed.

\section{CONCLUSION}

The resonance driving terms formalism was applied to betatron coupling in electron storage rings. Analytical formulas have been derived establishing a clear correlation between the different definitions of vertical emittance. On the wake of this, a correction scheme was conceived for coupling correction and vertical emittance minimization. The application of this scheme to the ESRF storage ring resulted in a record low vertical emittance of (4.4 \pm $0.7) \mathrm{pm}$, corresponding to an emittance ratio between the two transverse planes of about $0.1 \%$. Two procedures for the automatic compensation of coupling introduced by insertion devices were devised and successfully tested, providing a stable vertical emittance within 6 and $7 \mathrm{pm}$ during beam delivery.

\section{ACKNOWLEDGMENTS}

We are grateful to the ESRF Operation Group for its support in preparing and carrying out measurements, and for its help in improving the correction software and procedures.

\section{APPENDIX A: RMS TRANSVERSE EMITTANCES AND TWISS PARAMETERS IN PRESENCE OF COUPLING, DESCRIPTION IN THE RDT FORMALISM}

In this Appendix the single particle turn-by-turn motion, represented by the complex Courant-Snyder coordinate $h_{r}(N)=\tilde{r}(N)-i \tilde{p}_{r}(N)$, where $r$ stands for either $x$ or $y$, with $N$ the number of turns, is described in terms of coupling RDTs and normal form coordinates [16,27]:

$$
\zeta_{r}^{ \pm}(N)=\sqrt{2 I_{r}} e^{\mp i\left(2 \pi N Q_{r}+\psi_{r}\right)},
$$

where $2 I_{r}$ and $\psi_{r}$ are action-and-phase coordinates. In the linear case $2 I_{r}$ reduce to the linear Courant-Snyder singleparticle invariants. With coupling they correspond to the two eigenmode invariants, while in the presence of nonlinearities, they provide the nonlinear invariants.

Electron beams experiencing radiation damping lose memory of their initial parameters to reach, after a transient phase, and equilibrium state between radiation damping and diffusion. It can be argued that the use of the Lie algebra and RDT formalism of Refs. [18-20] is not legitimate, these two processes not being Hamiltonian (the betatron phase space volume is not preserved). The use of the normal form single-particle invariants $\sqrt{2 I_{r}}$ may be in contradiction with the fact that under radiation damping and diffusion, such invariants are not well defined, even though their RMS values are in equilibrium, hence invariant. A practical way out may be found with the following consideration: even though $\sqrt{2 I_{r}}$ may not be considered as integrals of motion, they can be considered so within a temporal window of several turns, both the effects of radiation and diffusion being slow compared to the betatron motion (damping time at the ESRF storage ring is $7 \mathrm{~ms}$, corresponding to about 2500 turns, and each turn the beam executes tens of betatron oscillations, the tunes being $Q_{x}=36.44$ and $Q_{y}=13.39$ ). Therefore the RMS equilibrium emittances $\mathcal{E}_{u, v}$ may be computed assuming an arbitrary stationary distribution.

The scope of this Appendix is the derivation of the RMS apparent and projected emittances as functions of the equilibrium emittances and the coupling RDTs. The evaluation of all second-order moments allow the derivation of analytical expressions relating the eigen-Twiss parameters 
$\beta_{i j}, \alpha_{i j}$, and $\gamma_{i j}$ to the uncoupled Twiss parameters and the RDTs.

The starting point is the betatron single particle turn-byturn motion derived in Ref. [20]:

$$
\begin{aligned}
h_{x}(N)= & \cosh (2 \mathcal{P}) \zeta_{x}^{-}(N) \\
& -i \sinh (2 \mathcal{P})\left[\frac{f_{1001}}{\mathcal{P}} \zeta_{y}^{-}(N)+\frac{f_{1010}}{\mathcal{P}} \zeta_{y}^{+}(N)\right] \\
h_{y}(N)= & \cosh (2 \mathcal{P}) \zeta_{y}^{-}(N) \\
& -i \sinh (2 \mathcal{P})\left[\frac{f_{1001}^{*}}{\mathcal{P}} \zeta_{x}^{-}(N)+\frac{f_{1010}}{\mathcal{P}} \zeta_{x}^{+}(N)\right],
\end{aligned}
$$

where $\mathcal{P}, f_{1001}$, and $f_{1010}$ have been already introduced in Eqs. (9) and (10). As stated in the Introduction, dispersion contributions are assumed to be already subtracted, leaving pure betatron coordinates. By inserting the definitions of $\mathcal{C}$ and $\mathcal{S}_{ \pm}$of Eqs. (6)-(8), the above equations may be rewritten as

$$
\begin{aligned}
h_{x}(N) & =\tilde{x}(N)-i \tilde{p}_{x}(N) \\
& =\mathcal{C} \zeta_{x}^{-}(N)-i \mathcal{S}_{-} e^{i q_{-}} \zeta_{y}^{-}(N)-i \mathcal{S}_{+} e^{i q_{+}} \zeta_{y}^{+}(N) \\
h_{y}(N) & =\tilde{y}(N)-i \tilde{p}_{y}(N) \\
& =\mathcal{C} \zeta_{y}^{-}(N)-i \mathcal{S}_{-} e^{-i q_{-}} \zeta_{x}^{-}(N)-i \mathcal{S}_{+} e^{i q_{+}} \zeta_{x}^{+}(N) .
\end{aligned}
$$

The above system needs to be rewritten in terms of the real Cartesian coordinates. The following relations and definitions are used:

$$
\begin{aligned}
\zeta_{r}^{ \pm} & =\sqrt{2 I_{r}} e^{\mp i\left(2 \pi N Q_{r}+\psi_{r}\right)}=\sqrt{2 I_{r}}\left[\cos \left(\tau_{r}\right) \mp i \sin \left(\tau_{r}\right)\right], \\
\tau_{r} & =2 \pi N Q_{r}+\psi_{r} .
\end{aligned}
$$

$r$ stands as usual for either $x$ or $y$. The real Courant-Snyder coordinates then read

$$
\begin{aligned}
\tilde{x}= & \mathcal{C} \sqrt{2 I_{x}} \cos \left(\tau_{x}\right)+\sqrt{2 I_{y}}\left[\mathcal{S}_{-} \sin \left(\tau_{y}+q_{-}\right)\right. \\
& \left.-\mathcal{S}_{+} \sin \left(\tau_{y}-q_{+}\right)\right] \\
\tilde{p}_{x}= & -\mathcal{C} \sqrt{2 I_{x}} \sin \left(\tau_{x}\right)+\sqrt{2 I_{y}}\left[\mathcal{S}_{-} \cos \left(\tau_{y}+q_{-}\right)\right. \\
& \left.+\mathcal{S}_{+} \cos \left(\tau_{y}-q_{+}\right)\right], \\
\tilde{y}= & \mathcal{C} \sqrt{2 I_{y}} \cos \left(\tau_{y}\right)+\sqrt{2 I_{x}}\left[\mathcal{S}_{-} \sin \left(\tau_{x}-q_{-}\right)\right. \\
& \left.-\mathcal{S}_{+} \sin \left(\tau_{x}-q_{+}\right)\right] \\
\tilde{p}_{y}= & -\mathcal{C} \sqrt{2 I_{y}} \sin \left(\tau_{y}\right)+\sqrt{2 I_{x}}\left[\mathcal{S}_{-} \cos \left(\tau_{x}-q_{-}\right)\right. \\
& \left.+\mathcal{S}_{+} \cos \left(\tau_{x}-q_{+}\right)\right] .
\end{aligned}
$$

Being the Courant-Snyder transformation symplectic, apparent and projected emittances can be evaluated in this coordinate system, being

$$
\epsilon_{r}^{2}=\sigma_{r} \sigma_{p}-\sigma_{r p}^{2}=\tilde{\sigma}_{r} \tilde{\sigma}_{p}-\tilde{\sigma}_{r p}^{2}
$$

$$
\mathbb{E}_{r}=\tilde{\sigma}_{r}^{2}
$$

The mean value of a generic function $F\left(I_{x}, I_{y}, \psi_{x}, \psi_{y}\right)$ is computed by integrating its product with the particle distribution function, $\rho\left(I_{x}, I, y, \psi_{x}, \psi_{y}\right)$. By making use of the physical constraints imposed by the equilibrium state, and hence by the matching condition, $\rho$ may not depend on the phases (i.e. all phases are equally probable). Being the two normal form variables representatives of eigenmodes, $\rho$ may be decomposed as the product of two independent distribution, $\rho\left(I_{x}, I_{y}\right)=\rho_{x}\left(I_{x}\right) \times \rho_{y}\left(I_{y}\right)$. This property makes it possible to ignore all integrands $\propto$ $\sqrt{2 I_{x} 2 I_{y}} e^{i \pm\left(\psi_{x} \pm \psi_{y}\right)}$, i.e.,

$$
\left\langle g\left(\psi_{x}, \psi_{y}\right) \sqrt{\left(2 I_{x}\right)\left(2 I_{y}\right)} e^{i \pm\left(\psi_{x} \pm \psi_{y}\right)}\right\rangle=0,
$$

where $g$ is an arbitrary function.

In computing the products of the Courant-Snyder coordinates, several trigonometric terms appear. However, only few contribute to the RMS values when averaging over the phases $\psi_{r}$, i.e., over $\tau_{r}$, the $2 \pi N Q_{r}$ being only a constant term, that is transparent to the integral. The following relations apply and are used in the next computations (even if not made explicit, all integrals are defined over a limited range: $\psi_{r} \in[0,2 \pi]$ and $\left.I_{r} \in\left[0, I_{r, \max }\right]\right)$ :

$$
\begin{gathered}
\langle F\rangle=\frac{1}{(2 \pi)^{2} A_{u} A_{v}} \\
\times \int F\left(I_{x}, I_{y}, \psi_{x}, \psi_{y}\right) \rho_{x}\left(I_{x}\right) \rho_{y}\left(I_{y}\right) d \psi_{x} d \psi_{y} d I_{x} d I_{y}, \\
2 \mathcal{E}_{u, v}=\frac{1}{A_{u, v}} \int\left(2 I_{x, y}\right) \rho_{x, y} d I_{x, y}, \quad A_{u, v}=\int \rho_{x, y} d I_{x, y}, \\
0=\int \sin \left(M \tau_{r}\right) d \tau_{r}=\int \cos \left(M \tau_{r}\right) d \tau_{r}, \quad M \in \mathbb{Z} \neq 0 \\
\pi=\int \sin ^{2}\left(\tau_{r}\right) d \tau_{r}=\int \cos ^{2}\left(\tau_{r}\right) d \tau_{r},
\end{gathered}
$$

where $\mathcal{E}_{u, v}$ are the equilibrium emittances. Other trigonometric terms whose mean values differ from zero are

$$
\begin{aligned}
& \left\langle\sin \left(\tau_{r}+q_{ \pm}\right) \sin \left(\tau_{r}+q_{\mp}\right)\right\rangle=\pi \cos \left(q_{ \pm}-q_{\bar{\mp}}\right), \\
& \left\langle\cos \left(\tau_{r}+q_{ \pm}\right) \cos \left(\tau_{r}+q_{\mp}\right)\right\rangle=\pi \cos \left(q_{ \pm}-q_{\mp}\right), \\
& \left\langle\sin \left(\tau_{r}+q_{ \pm}\right) \cos \left(\tau_{r}+q_{\mp}\right)\right\rangle=\pi \sin \left(q_{ \pm}-q_{\mp}\right) .
\end{aligned}
$$

By making use of the above definitions and properties, the second-order moments of Eqs. (A5) and (A6) can be computed. The final result is 


$$
\begin{aligned}
\tilde{\sigma}_{x}^{2} & =\mathcal{C}^{2} \mathcal{E}_{u}+\left[\mathcal{S}_{-}^{2}+\mathcal{S}_{+}^{2}-2 \mathcal{S}_{-} \mathcal{S}_{+} \cos \left(q_{+}+q_{-}\right)\right] \mathcal{E}_{v}, \\
\tilde{\sigma}_{y}^{2} & =\left[\mathcal{S}_{-}^{2}+\mathcal{S}_{+}^{2}-2 \mathcal{S}_{-} \mathcal{S}_{+} \cos \left(q_{+}-q_{-}\right)\right] \mathcal{E}_{u}+\mathcal{C}^{2} \mathcal{E}_{v}, \\
\tilde{\sigma}_{p_{x}}^{2} & =\mathcal{C}^{2} \mathcal{E}_{u}+\left[\mathcal{S}_{-}^{2}+\mathcal{S}_{+}^{2}+2 \mathcal{S}_{-} \mathcal{S}_{+} \cos \left(q_{+}+q_{-}\right)\right] \mathcal{E}_{v}, \\
\tilde{\sigma}_{p_{y}}^{2} & =\left[\mathcal{S}_{-}^{2}+\mathcal{S}_{+}^{2}+2 \mathcal{S}_{-} \mathcal{S}_{+} \cos \left(q_{+}-q_{-}\right)\right] \mathcal{E}_{u}+\mathcal{C}^{2} \mathcal{E}_{v}, \\
\tilde{\sigma}_{x p_{x}} & =\left[2 \mathcal{S}_{+} \mathcal{S}_{-} \sin \left(q_{+}+q_{-}\right)\right] \mathcal{E}_{v}, \\
\tilde{\sigma}_{y p_{y}} & =\left[2 \mathcal{S}_{+} \mathcal{S}_{-} \sin \left(q_{+}-q_{-}\right)\right] \mathcal{E}_{u}
\end{aligned}
$$

It is worthwhile noticing that unless either $\mathcal{S}_{-}$or $\mathcal{S}_{+}$is negligible as compared to the other, the equilibrium Courant-Snyder phase space is not a circle anymore, being $\tilde{\sigma}_{r} \neq \tilde{\sigma}_{p}, \tilde{\sigma}_{r p} \neq 0$. This is equivalent to the fact that $\mathbb{E}_{r} \neq \epsilon_{r}$.

The projected emittances of Eq. (A7) eventually read

$$
\begin{aligned}
\epsilon_{x} & =\sqrt{\left(\mathcal{C}^{2} \mathcal{E}_{u}+\left[\mathcal{S}_{-}^{2}+\mathcal{S}_{+}^{2}\right] \mathcal{E}_{v}\right)^{2}-\left(2 \mathcal{S}_{+} \mathcal{S}_{-} \mathcal{E}_{v}\right)^{2}}, \\
\epsilon_{y} & =\sqrt{\left(\mathcal{C}^{2} \mathcal{E}_{v}+\left[\mathcal{S}_{-}^{2}+\mathcal{S}_{+}^{2}\right] \mathcal{E}_{u}\right)^{2}-\left(2 \mathcal{S}_{+} \mathcal{S}_{-} \mathcal{E}_{u}\right)^{2}},
\end{aligned}
$$

proving Eqs. (13) and (14). The apparent emittances of Eqs. (4) and (5) instead are

$$
\begin{aligned}
\mathbb{E}_{x} & =\tilde{\sigma}_{x}^{2} \\
& =\mathcal{C}^{2} \mathcal{E}_{u}+\left[\mathcal{S}_{-}^{2}+\mathcal{S}_{+}^{2}-2 \mathcal{S}_{-} \mathcal{S}_{+} \cos \left(q_{+}+q_{-}\right)\right] \mathcal{E}_{v}, \\
\mathbb{E}_{y} & =\tilde{\sigma}_{y}^{2} \\
& =\mathcal{C}^{2} \mathcal{E}_{v}+\left[\mathcal{S}_{-}^{2}+\mathcal{S}_{+}^{2}-2 \mathcal{S}_{-} \mathcal{S}_{+} \cos \left(q_{+}-q_{-}\right)\right] \mathcal{E}_{u} .
\end{aligned}
$$

Formulas in Eq. (A17) may be expressed in Cartesian coordinates after the inverse Courant-Snyder transformation

$$
\vec{r}=\mathbf{W}_{\mathbf{r}}^{-1} \overrightarrow{\tilde{r}}, \rightarrow\left(\begin{array}{l}
r \\
p
\end{array}\right)=\left(\begin{array}{cc}
\left(\beta_{r}\right)^{1 / 2} & 0 \\
-\alpha_{r}\left(\beta_{r}\right)^{-1 / 2} & \left(\beta_{r}\right)^{-1 / 2}
\end{array}\right)\left(\begin{array}{c}
\tilde{r} \\
\tilde{p}
\end{array}\right),
$$

where the Twiss parameters are the one computed from the linear uncoupled lattice. The following relation between the second-order moments applies:

$$
\begin{gathered}
\sigma_{r}^{2}=\beta_{r} \tilde{\sigma}_{r}^{2} \\
\sigma_{p}^{2}=\frac{1}{\beta_{r}}\left[\alpha_{r}^{2} \tilde{\sigma}_{r}^{2}+\tilde{\sigma}_{p}^{2}-2 \alpha_{r} \tilde{\sigma}_{p r}\right] \\
\sigma_{p r}=-\alpha_{r} \tilde{\sigma}_{r}^{2}+\tilde{\sigma}_{p r} .
\end{gathered}
$$

After some algebra the same RMS moments may be rewritten as

$$
\begin{aligned}
\sigma_{x}^{2} & =\beta_{11} \mathcal{E}_{u}+\beta_{12} \mathcal{E}_{v}, \quad \sigma_{y}^{2}=\beta_{21} \mathcal{E}_{u}+\beta_{22} \mathcal{E}_{v}, \\
\sigma_{p_{x}}^{2} & =\gamma_{11} \mathcal{E}_{u}+\gamma_{12} \mathcal{E}_{v}, \quad \sigma_{p_{y}}^{2}=\gamma_{21} \mathcal{E}_{u}+\gamma_{22} \mathcal{E}_{v}, \\
\sigma_{x p_{x}} & =-\alpha_{11} \mathcal{E}_{u}+-\alpha_{12} \mathcal{E}_{v}, \quad \sigma_{y p_{y}}=-\alpha_{21} \mathcal{E}_{u}+-\alpha_{22} \mathcal{E}_{v},
\end{aligned}
$$

where the generalized coupled Twiss parameters read

$$
\begin{aligned}
\beta_{11}= & \beta_{x} \mathcal{C}^{2} \\
\beta_{12}= & \beta_{x}\left[\mathcal{S}_{-}^{2}+\mathcal{S}_{+}^{2}-2 \mathcal{S}_{-} \mathcal{S}_{+} \cos \left(q_{+}+q_{-}\right)\right] \\
\beta_{21}= & \beta_{y}\left[\mathcal{S}_{-}^{2}+\mathcal{S}_{+}^{2}-2 \mathcal{S}_{-} \mathcal{S}_{+} \cos \left(q_{+}-q_{-}\right)\right] \\
\beta_{22}= & \beta_{y} \mathcal{C}^{2}, \\
\alpha_{11}= & \alpha_{x} \mathcal{C}^{2} \\
\alpha_{12}= & \alpha_{x}\left[\mathcal{S}_{-}^{2}+\mathcal{S}_{+}^{2}-2 \mathcal{S}_{-} \mathcal{S}_{+} \cos \left(q_{+}+q_{-}\right)\right] \\
& -2 \mathcal{S}_{-} \mathcal{S}_{+} \sin \left(q_{+}+q_{-}\right) \\
\alpha_{21}= & \alpha_{y}\left[\mathcal{S}_{-}^{2}+\mathcal{S}_{+}^{2}-2 \mathcal{S}_{-} \mathcal{S}_{+} \cos \left(q_{+}-q_{-}\right)\right] \\
& -2 \mathcal{S}_{-} \mathcal{S}_{+} \sin \left(q_{+}-q_{-}\right) \\
\alpha_{22}= & \alpha_{y} \mathcal{C}^{2},
\end{aligned}
$$
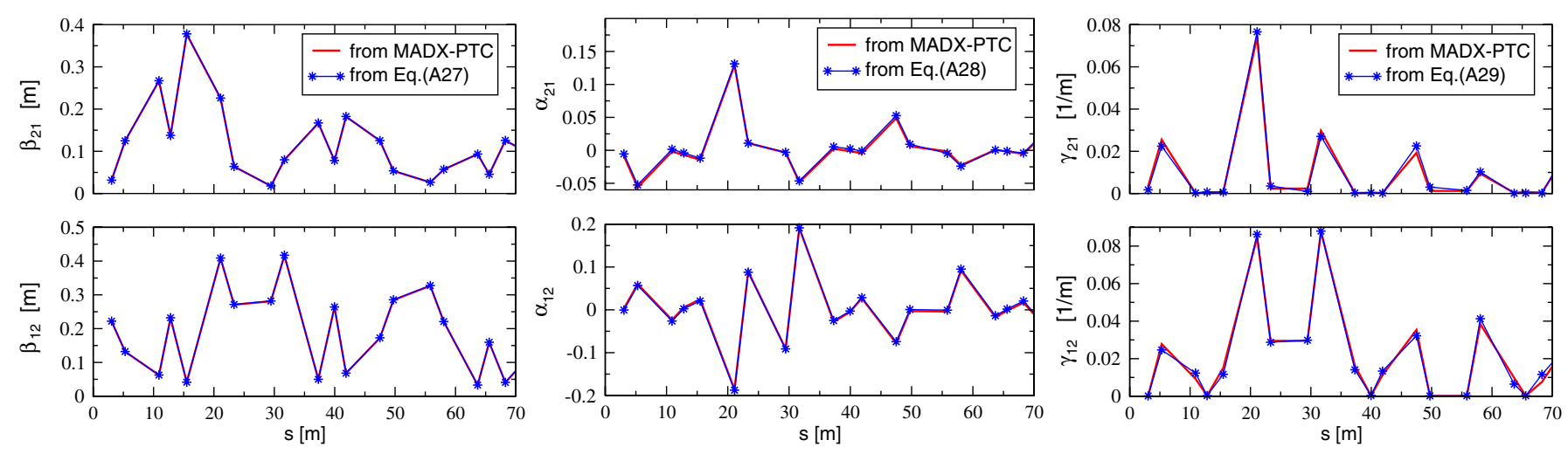

FIG. 11. Comparison between the coupled Twiss parameters as computed by MADX-PTC (Twiss module) and the ones from Eqs. (A27)-(A29) (zoom of the entire ring length of $844 \mathrm{~m}$ ). Parameters refer to the ESRF storage ring lattice with the same coupling of Fig. 1. The uncoupled Twiss parameters, $\beta, \alpha$, and $\gamma$, are obtained from the MADX by using the ideal uncoupled lattice. 


$$
\begin{aligned}
\gamma_{11}= & \gamma_{x} \mathcal{C}^{2} \\
\gamma_{12}= & \gamma_{x}\left[\mathcal{S}_{-}^{2}+\mathcal{S}_{+}^{2}+\frac{1-\alpha_{x}^{2}}{1+\alpha_{x}^{2}} 2 \mathcal{S}_{-} \mathcal{S}_{+} \cos \left(q_{+}+q_{-}\right)\right. \\
& \left.-\frac{4 \alpha_{x}}{1+\alpha_{x}^{2}} \mathcal{S}_{-} \mathcal{S}_{+} \sin \left(q_{+}+q_{-}\right)\right] \\
\gamma_{21}= & \gamma_{y}\left[\mathcal{S}_{-}^{2}+\mathcal{S}_{+}^{2}+\frac{1-\alpha_{y}^{2}}{1+\alpha_{y}^{2}} 2 \mathcal{S}_{-} \mathcal{S}_{+} \cos \left(q_{+}-q_{-}\right)\right. \\
& \left.-\frac{4 \alpha_{y}}{1+\alpha_{y}^{2}} \mathcal{S}_{-} \mathcal{S}_{+} \sin \left(q_{+}-q_{-}\right)\right] \\
\gamma_{22}= & \gamma_{y} \mathcal{C}^{2} .
\end{aligned}
$$

In a decoupled lattice $\left(\mathcal{C}=1, \mathcal{S}_{ \pm}=0\right)$ all 12 -and $21-$ parameters are zero, while those labeled with 11 and 22 correspond to the horizontal and vertical ones, respectively. Codes such as MADX-PTC are capable to compute the generalized Twiss parameters. A comparison between the latter and the ones computed via Eqs. (A27)-(A29) is shown in Fig. 11 (the ESRF storage ring lattice is used with the same coupling of Fig. 1). The agreement is remarkable.

\section{APPENDIX B: EQUILIBRIUM TRANSVERSE EMITTANCES IN PRESENCE OF COUPLING: DESCRIPTION IN THE RDT FORMALISM}

Introduction and basic definitions.-In Eq. (A11) the equilibrium emittances $\mathcal{E}_{u, v}$ have been left only implicitly defined. The scope of this Appendix is the derivation of explicit analytic formulas for their evaluation in terms of the damping and diffusion terms, the dispersion invariants, and the coupling RDTs. To this end RDT formalism is merged to the one developed in Ref. [8], whose main definitions and results are here summarized for the sake of consistency.

The turn-by-turn evolution of a single particle is described by a $4 \mathrm{D}$ vector $\vec{z}=\left(x, p_{x}, y, p_{y}\right)$ (longitudinal motion is here assumed to be decoupled and ignored) and the one-turn map $\mathcal{M}(s)$ according to

$$
\vec{z}_{s+C}=\mathcal{M}(s) \vec{z}_{s},
$$

where $C$ is the circumference, and $s$ is the generic location at which the dynamics is observed. $\mathcal{M}(s)$ is symplectic and its eigenvectors $\vec{v}_{ \pm 1, \pm 2}$, properly normalized (details can be found in Ref. [8]), may be used to define a symplectic matrix $\mathcal{U}$, such that

$$
\begin{aligned}
\mathcal{M} & =\mathcal{U} e^{\Lambda} \mathcal{U}^{-1} \\
e^{\Lambda} & =\left(\begin{array}{cccc}
e^{i Q u} & 0 & 0 & 0 \\
0 & e^{-i Q u} & 0 & 0 \\
0 & 0 & e^{i Q v} & 0 \\
0 & 0 & 0 & e^{-i Q v}
\end{array}\right) .
\end{aligned}
$$

Two invariant matrices defined as follows:

$$
\begin{gathered}
G_{i}=-J\left(\vec{v}_{i}^{*} \vec{v}_{i}^{T}+\vec{v}_{i} \vec{v}_{i}^{\dagger}\right) J, \quad i=1,2 \\
J=\left(\begin{array}{ccccc}
0 & 1 & 0 & 0 \\
-1 & 0 & 0 & 0 \\
0 & 0 & 0 & 1 \\
0 & 0 & -1 & 0
\end{array}\right)
\end{gathered}
$$

determine the covariance matrix (i.e. the second-order moments) $\Sigma_{i j}=\left\langle z_{i} z_{j}\right\rangle$, according to

$$
\begin{aligned}
\Sigma & =\Sigma_{u} \mathcal{E}_{u}+\Sigma_{v} \mathcal{E}_{v}, \quad \Sigma_{u}=-J G_{1} J, \\
\Sigma_{v} & =-J G_{2} J .
\end{aligned}
$$

The diffusion integrals $\bar{d}_{i}$ read

$$
\bar{d}_{i}=\oint \operatorname{Tr}\left[G_{i} D_{\beta}\right] d s, \quad i=1,2, \quad D_{\beta}=\mathcal{B} D_{s} \mathcal{B}^{T},
$$

where the diffusion and dispersion matrices, $D_{s}$ and $\mathcal{B}$, respectively, are so defined:

$$
\begin{gathered}
D_{s}=\left(\begin{array}{cccccc}
0 & 0 & 0 & 0 & 0 & 0 \\
0 & 0 & 0 & 0 & 0 & 0 \\
0 & 0 & 0 & 0 & 0 & 0 \\
0 & 0 & 0 & 0 & 0 & 0 \\
0 & 0 & 0 & 0 & 0 & 0 \\
0 & 0 & 0 & 0 & 0 & \mathrm{~d}(s)
\end{array}\right), \\
\mathcal{B}=\left(\begin{array}{ccccccc}
1 & 0 & 0 & 0 & 0 & -D_{x} \\
0 & 1 & 0 & 0 & 0 & -D_{x}^{\prime} \\
0 & 0 & 1 & 0 & 0 & -D_{y} \\
0 & 0 & 0 & 1 & 0 & -D_{y}^{\prime} \\
-D_{x} & -D_{x}^{\prime} & -D_{y} & -D_{y}^{\prime} & 1 & 0 \\
0 & 0 & 0 & 0 & 0 & 1
\end{array}\right) .
\end{gathered}
$$

Even though $D_{\beta}$ needs to be a $6 \times 6$ matrix including longitudinal dispersive terms, only the upper left $4 \times 4$ block enters in the product $G_{i} D_{\beta}$ of Eq. (B6). $\mathrm{d}(s)$ is the same of Eq. (20).

The two damping integrals can be expressed in terms of $\mathcal{U}$ and of the damping matrix $B$, according to

$\chi_{1}=\oint\left[A_{11}+A_{22}\right] d s, \quad \chi_{2}=\oint\left[A_{33}+A_{44}\right] d s$,

where

$$
A=\mathcal{U}^{-1} B_{\beta} \mathcal{U}, \quad B_{\beta}=\mathcal{B} B \mathcal{B}^{-1},
$$




$$
B=\left(\begin{array}{cccccc}
0 & 0 & 0 & 0 & 0 & 0 \\
0 & \mathrm{~b}_{\mathrm{RF}} & 0 & 0 & 0 & 0 \\
0 & 0 & 0 & 0 & 0 & 0 \\
0 & 0 & 0 & \mathrm{~b}_{\mathrm{RF}} & 0 & 0 \\
0 & 0 & 0 & 0 & 0 & 0 \\
\mathrm{~b}_{\delta x} & 0 & \mathrm{~b}_{\delta y} & 0 & 0 & \mathrm{~b}_{z}
\end{array}\right) .
$$

The damping coefficients $b_{\text {rf }}$ and $b_{\delta r}$ are the same of Eqs. (22) and (23), while $\mathrm{b}_{z}=P_{\gamma} c P_{0}$. As for diffusion, only the first $4 \times 4$ block of $B_{\beta}$ will be used in the computation of $A$. The transverse equilibrium emittances are eventually given by

$$
\mathcal{E}_{u}=\frac{1}{2} \frac{\bar{d}_{1}}{\chi_{1}}, \quad \text { and } \quad \mathcal{E}_{v}=\frac{1}{2} \frac{\bar{d}_{2}}{\chi_{2}} .
$$

$\mathcal{U}$ matrix and RDTs. - The connection between coupling RDTs and the above relations is carried out by making explicit the matrix $\mathcal{U}$ of Eq. (B2). To this end the system of Eq. (A3), evaluated at $N=1$, can be written as

$$
\vec{h}=F^{-1} e^{i \Lambda} \vec{\zeta}_{0},
$$

where $\vec{h}=\left(h_{x}, h_{x}^{*}, h_{y}, h_{y}^{*}\right), \vec{\zeta}_{0}=\left(\zeta_{x 0}^{+}, \zeta_{x 0}^{-}, \zeta_{y 0}^{+}, \zeta_{y 0}^{-}\right)$, with $\zeta_{r 0}^{ \pm}=\sqrt{2 I_{r}} e^{\mp i \psi_{r 0}}(r$ stands for either $x$ or $y)$. The (complex) matrix $F^{-1}$ instead reads

$$
F^{-1}=\left(\begin{array}{cccc}
\mathcal{C} & 0 & -i \mathcal{S}_{-} e^{i q_{-}} & -i \mathcal{S}_{+} e^{i q_{+}} \\
0 & \mathcal{C}^{*} & i \mathcal{S}_{+}^{*} e^{-i q_{+}} & +i \mathcal{S}_{-}^{*} e^{-i q_{-}} \\
-i \mathcal{S}_{-} e^{-i q_{-}} & -i \mathcal{S}_{+} e^{i q_{+}} & \mathcal{C} & 0 \\
i \mathcal{S}_{+}^{*} e^{-i q_{+}} & i \mathcal{S}_{-}^{*} e^{i q_{-}} & 0 & \mathcal{C}^{*}
\end{array}\right)
$$

$\vec{\zeta}_{0}$ can be expressed in terms of $\vec{h}_{0}$ by inverting the same system of Eq. (B13) at $N=0$, yielding to

$$
\vec{z}_{0}=F \vec{h}_{0}
$$

The complex Courant-Snyder coordinates $\vec{h}$ are obtained from the Cartesian ones of $\vec{z}$ via the following (nonsymplectic) transformation:

$$
\vec{h}=P W \vec{z}, \quad P=\left(\begin{array}{cc}
\mathbf{P}_{\mathbf{c}} & \mathbf{0} \\
\mathbf{0} & \mathbf{P}_{\mathbf{c}}
\end{array}\right), \quad W=\left(\begin{array}{cc}
\mathbf{W}_{\mathbf{x}} & \mathbf{0} \\
\mathbf{0} & \mathbf{W}_{\mathbf{y}}
\end{array}\right) .
$$

$\mathbf{W}_{\mathbf{r}}$ are the same $2 \times 2$ matrix of Eq. (A22), whereas the $4 \times 4$ matrix $\mathbf{P}$ transforms the real Courant-Snyder coordinates $(\tilde{r}, \tilde{p})$ in the complex set $\left(h_{r}, h_{r}^{*}\right)$, the $2 \times 2$ block $\mathbf{P}_{\mathbf{c}}$ reading

$$
\mathbf{P}_{\mathbf{c}}=\left(\begin{array}{cc}
1 & -i \\
1 & i
\end{array}\right)
$$

By inserting Eqs. (B16) and (B15) in Eq. (B13), the system reads

$$
\vec{z}=(F P W)^{-1} e^{i \Lambda}(F P W) \vec{z}_{0}, \Rightarrow \mathcal{U}=(F P W)^{-1},
$$

where the last equation follows the definition of $\mathcal{U}$ given in Eq. (B2). It is however more convenient to perform all calculations in the Courant-Snyder coordinates (with Twiss parameters from the ideal, uncoupled lattice)

$$
\overrightarrow{\tilde{z}}=(F P)^{-1} e^{i \Lambda}(F P) \overrightarrow{\tilde{z}}_{0}, \Rightarrow \tilde{U}=(F P)^{-1} .
$$

The reason is twofold. First, both the diffusion and damping integrals of Eqs. (B6) and (B9) apply on matrix traces, that are invariant under symplectic transformations, such as the Courant-Snyder. Second, the evaluation of the covariance matrices $\Sigma_{u, v}$ of Eq. (B5) in these coordinates will provide directly another analytical derivation of the generalized Twiss parameters. The matrix $\tilde{U}$ reads

$$
\tilde{U}=\frac{1}{2 i}\left(\begin{array}{cccc}
i \mathcal{C} & i \mathcal{C}^{*} & U_{3} & -i U_{3}^{*} \\
-\mathcal{C} & \mathcal{C}^{*} & i U_{4} & i U_{4}^{*} \\
U_{1} & -U_{1}^{*} & i \mathcal{C} & i \mathcal{C}^{*} \\
i U_{2} & i U_{2}^{*} & -\mathcal{C} & \mathcal{C}^{*}
\end{array}\right)
$$

where

$$
\begin{aligned}
& U_{1}=\mathcal{S}_{-} e^{-i q_{-}}-\mathcal{S}_{+}^{*} e^{-i q_{+}} \\
& U_{2}=\mathcal{S}_{-} e^{-i q_{-}}+\mathcal{S}_{+}^{*} e^{-i q_{+}} \\
& U_{3}=\mathcal{S}_{-} e^{+i q_{-}}-\mathcal{S}_{+}^{*} e^{-i q_{+}} \\
& U_{4}=\mathcal{S}_{-} e^{+i q_{-}}+\mathcal{S}_{+}^{*} e^{-i q_{+}} .
\end{aligned}
$$

It can be shown that $\left(\tilde{U} e^{i \Lambda} \tilde{U}^{-1}\right)$ is a $4 \times 4$ real symplectic matrix. The definition of $\tilde{U}$ given in Eq. (B20) requires however a further transformation. Indeed, in Ref. [8] it is requested that the matrix is built from four $4 \mathrm{D}$ column eigenvectors of $\mathcal{M}, \vec{v}_{ \pm 1, \pm 2}$, properly normalized, in the form

$$
\mathcal{U}=\left(\vec{v}_{1} \vec{v}_{-1} \vec{v}_{2} \vec{v}_{-2}\right), \quad \text { where } \vec{v}_{-i}=i \vec{v}_{i}^{*}
$$

In order to satisfy the above structure and the requested normalization, the following matrix is obtained:

$$
\tilde{\mathcal{U}}=\frac{1}{\sqrt{2}}\left(\begin{array}{cccc}
i \mathcal{C} & \mathcal{C}^{*} & U_{3} & i U_{3}^{*} \\
-\mathcal{C} & -i \mathcal{C}^{*} & i U_{4} & U_{4}^{*} \\
U_{1} & i U_{1}^{*} & i \mathcal{C} & \mathcal{C}^{*} \\
i U_{2} & U_{2}^{*} & -\mathcal{C} & -i \mathcal{C}^{*}
\end{array}\right)
$$

It can be shown that $\tilde{\mathcal{U}}$ is symplectic and that is derived from $\tilde{U}$ after the following transformation:

$$
\tilde{U}=\tilde{U} A, \quad A=\sqrt{2}\left(\begin{array}{cccc}
i & 0 & 0 & 0 \\
0 & 1 & 0 & 0 \\
0 & 0 & i & 0 \\
0 & 0 & 0 & 1
\end{array}\right) .
$$

It is straightforward to prove that $\left(\tilde{U} e^{i \Lambda} \tilde{U}^{-1}\right)=$ $\left(\tilde{\mathcal{U}} e^{i \Lambda} \tilde{\mathcal{U}}^{-1}\right)$ and hence that

$$
\overrightarrow{\tilde{z}}=\tilde{U} e^{i \Lambda} \tilde{U}^{-1} \overrightarrow{\tilde{z}}_{0},
$$


where $\overrightarrow{\tilde{z}}=\left(\tilde{x}, \tilde{p}_{x}, \tilde{y}, \tilde{p}_{y}\right)$ is the $4 \mathrm{D}$ coordinate vector in Courant-Snyder.

Generalized coupled Twiss parameters.-The matrix $\tilde{U}$ of Eq. (B23) and its two eigenvectors $\vec{v}_{1}=$ $1 / \sqrt{2}\left(i \mathcal{C},-\mathcal{C}, U_{1}, i U_{2}\right)$ and $\vec{v}_{2}=1 / \sqrt{2}\left(U_{3}, i U_{4}, i \mathcal{C},-\mathcal{C}\right)$ may now be used to derive the two (real and symmetric) invariant matrices in Courant-Snyder from Eq. (B3), yielding

$$
\tilde{G}_{1}=\left(\begin{array}{cccc}
\mathcal{C}^{2} & 0 & -\mathcal{C} \Re\left\{i U_{2}\right\} & \mathcal{C} \Re\left\{U_{1}\right\} \\
0 & \mathcal{C}^{2} & -\mathcal{C} \Re\left\{U_{2}\right\} & -\mathcal{C} \Re\left\{i U_{1}\right\} \\
-\mathcal{C} \Re\left\{i U_{2}\right\} & -\mathcal{C} \Re\left\{U_{2}\right\} & \left|U_{2}\right|^{2} & -\mathfrak{R}\left\{i U_{1}^{*} U_{2}\right\} \\
\mathcal{C} \Re\left\{U_{1}\right\} & -\mathcal{C} \Re\left\{i U_{1}\right\} & -\mathfrak{R}\left\{i U_{1}^{*} U_{2}\right\} & \left|U_{1}\right|^{2}
\end{array}\right)
$$

$\tilde{G}_{2}=\left(\begin{array}{cccc}\left|U_{4}\right|^{2} & -\mathfrak{R}\left\{i U_{3}^{*} U_{4}\right\} & \mathcal{C} \Re\left\{i U_{4}^{*}\right\} & -\mathcal{C} \Re\left\{U_{4}\right\} \\ -\mathfrak{R}\left\{i U_{3}^{*} U_{4}\right\} & \left|U_{3}\right|^{2} & \mathcal{C} \Re\left\{U_{3}\right\} & \mathcal{C} \Re\left\{i U_{3}^{*}\right\} \\ \mathcal{C} \Re\left\{i U_{4}^{*}\right\} & \mathcal{C} \Re\left\{U_{3}\right\} & \mathcal{C}^{2} & 0 \\ -\mathcal{C} \Re\left\{U_{4}\right\} & \mathcal{C} \Re\left\{i U_{3}^{*}\right\} & 0 & \mathcal{C}^{2}\end{array}\right)$,

where $\Re\{\cdot\}$ denotes the real part. The covariant matrices are inferred from $\tilde{G}_{1,2}$ according Eq. (B5) and from the inverse Courant-Snyder transformation $\left[\overrightarrow{\tilde{z}}^{T} \tilde{\Sigma}^{-1} \overrightarrow{\tilde{z}}=\right.$ $\vec{z}^{T}\left(W^{T} \tilde{\Sigma}^{-1} W\right) \vec{z} \Rightarrow \Sigma^{-1}=W^{T} \tilde{\Sigma}^{-1} W \Rightarrow \Sigma=$ $\left.W^{-1} \tilde{\Sigma}\left(W^{-1}\right)^{T}\right]$

$$
\tilde{\Sigma}_{u, v}=-J \tilde{G}_{1,2} J \Rightarrow \Sigma_{u, v}=-W^{-1} J \tilde{G}_{1,2} J\left(W^{-1}\right)^{T},
$$

where $W^{-1}$ is the inverse matrix of Eq. (B16) and $\left(W^{-1}\right)^{T}$ denotes its transpose. The evaluation of $\Sigma_{u, v}$ in Eq. (B28) provides the same generalized coupled Twiss parameters of Eqs. (A27)-(A29), after noting that

$$
\begin{aligned}
& \Sigma_{u}=\left(\begin{array}{cccc}
\beta_{11} & -\alpha_{11} & \boldsymbol{\Sigma}_{\mathbf{u v}} \\
-\alpha_{11} & \gamma_{11} & \beta_{21} & -\alpha_{21} \\
\boldsymbol{\Sigma}_{\mathbf{u v}} & -\alpha_{21} & \gamma_{21}
\end{array}\right), \\
& \Sigma_{v}=\left(\begin{array}{cccc}
\beta_{12} & -\alpha_{12} & \boldsymbol{\Sigma}_{\mathbf{v u}} \\
-\alpha_{12} & \gamma_{12} & \beta_{22} & -\alpha_{22} \\
\mathbf{\Sigma}_{\mathbf{v u}} & -\alpha_{22} & \gamma_{22}
\end{array}\right),
\end{aligned}
$$

where $\boldsymbol{\Sigma}_{\mathbf{u v}}$ and $\boldsymbol{\Sigma}_{\mathbf{v u}}$ are $2 \times 2$ blocks.

Diffusion integrals. - The diffusion integrals are obtained from Eq. (B6) after evaluating

$\operatorname{Tr}\left[G_{i} D_{\beta}\right]=\operatorname{Tr}\left[\tilde{G}_{i} \tilde{D}_{\beta}\right]=, i=1,2, \quad \tilde{D}_{\beta}=\tilde{\mathcal{B}} D_{s} \tilde{\mathcal{B}}^{T}$.

Since $\vec{z}_{\beta}=\mathcal{B} \vec{z}$ and $\overrightarrow{\tilde{z}}=W \vec{z}, \tilde{\mathcal{B}}=W \mathcal{B}$, i.e.,

$$
\tilde{\mathcal{B}}=\left(\begin{array}{cccccc}
1 / \sqrt{\beta_{x}} & 0 & 0 & 0 & 0 & -\tilde{D}_{x} \\
\alpha_{x} / \sqrt{\beta_{x}} & \sqrt{\beta_{x}} & 0 & 0 & 0 & -\tilde{D}_{x}^{\prime} \\
0 & 0 & 1 / \sqrt{\beta_{y}} & 0 & 0 & -\tilde{D}_{y} \\
0 & 0 & \alpha_{y} / \sqrt{\beta_{y}} & \sqrt{\beta_{y}} & 0 & -\tilde{D}_{y}^{\prime} \\
-\tilde{D}_{x} & -\tilde{D}_{x}^{\prime} & -\tilde{D}_{y} & -\tilde{D}_{y}^{\prime} & 1 & 0 \\
0 & 0 & 0 & 0 & 0 & 1
\end{array}\right),
$$

where $\tilde{D}_{r}$ and $\tilde{D}_{r}^{\prime}$ are the dispersion functions in CourantSnyder and $\mathcal{H}_{r}=\tilde{D}_{r}^{2}+\tilde{D}_{r}^{\prime 2}$. The evaluation of Eq. (B31) yields

$\operatorname{Tr}\left[G_{1} D_{\beta}\right]=-\mathrm{d}(s)\left\{\mathcal{C}^{2} \mathcal{H}_{x}^{2}+\left[\mathcal{S}_{-}^{2}+\mathcal{S}_{+}^{2}\right] \mathcal{H}_{y}^{2}+R_{1}\right\}$

$\operatorname{Tr}\left[G_{2} D_{\beta}\right]=-\mathrm{d}(s)\left\{\mathcal{C}^{2} \mathcal{H}_{y}^{2}+\left[\mathcal{S}_{-}^{2}+\mathcal{S}_{+}^{2}\right] \mathcal{H}_{x}^{2}+R_{2}\right\}$,

where the terms $R_{1,2}$ oscillate with $q_{ \pm}$and are assumed to have a negligible contribute to the diffusion integrals, which eventually read

$\bar{d}_{1}=\oint \mathrm{d}(s)\left\{\mathcal{C}^{2} \mathcal{H}_{x}^{2}+\left[\mathcal{S}_{-}^{2}+\mathcal{S}_{+}^{2}\right] \mathcal{H}_{y}^{2}\right\} d s$

$\bar{d}_{2}=\oint \mathrm{d}(s)\left\{\mathcal{C}^{2} \mathcal{H}_{y}^{2}+\left[\mathcal{S}_{-}^{2}+\mathcal{S}_{+}^{2}\right] \mathcal{H}_{x}^{2}\right\} d s$,

where $\mathrm{d}(s)$ has been already introduced in Eq. (20). It is worthwhile noticing that the sum rules for the diffusion integrals are easily retrieved from Eqs. (B33) and (12) in two extreme cases:

$\bar{d}_{1}+\bar{d}_{2}=$ const for $\mathcal{S}_{+}^{2} \ll \mathcal{S}_{-}^{2}$ (diff resonance),

$\bar{d}_{1}-\bar{d}_{2}=$ const for $\mathcal{S}_{-}^{2} \ll \mathcal{S}_{+}^{2}$ (sum resonance).

Damping integrals.-The damping integrals are derived from Eqs. (B8)-(B11). In Eq. (B10) $\mathcal{U}$ needs to be replaced by $\tilde{U}$ to account for the Courant-Snyder transformation. Equation (B10) then reads

$$
A=\tilde{\mathcal{U}}^{-1} B_{\beta} \tilde{\mathcal{U}}
$$

where $\tilde{U}$ has been derived in Eq. (B23). Only the upper left $4 \times 4$ block of the $6 \times 6$ matrix $B_{\beta}$ is considered in the products with the $4 \times 4$ matrix $\tilde{U}$, synchrobetatron coupling not being included here. Only the four diagonal elements of $A$ are of interest for the damping integrals. Their sums read

$$
\begin{aligned}
A_{11}+A_{22}= & \mathcal{C}^{2} \mathrm{~b}_{\mathrm{RF}}+\left[\mathcal{S}_{-}^{2}-\mathcal{S}_{+}^{2}\right] \mathrm{b}_{\mathrm{RF}}-\mathcal{C}^{2} D_{x} \mathrm{~b}_{\delta x} \\
& -\left[\mathcal{S}_{-}^{2}-\mathcal{S}_{+}^{2}\right] D_{y} \mathrm{~b}_{\delta y}+R_{3} \\
A_{22}+A_{33}= & \mathcal{C}^{2} \mathrm{~b}_{\mathrm{RF}}+\left[\mathcal{S}_{-}^{2}-\mathcal{S}_{+}^{2}\right] \mathrm{b}_{\mathrm{RF}}-\mathcal{C}^{2} D_{y} \mathrm{~b}_{\delta y} \\
& -\left[\mathcal{S}_{-}^{2}-\mathcal{S}_{+}^{2}\right] D_{x} \mathrm{~b}_{\delta x}+R_{4},
\end{aligned}
$$

where the terms $R_{3,4}$ oscillate with $q_{ \pm}$and are assumed to have a negligible contribute to the diffusion integrals. 
Since $1=\mathcal{C}^{2}+\mathcal{S}_{-}^{2}-\mathcal{S}_{+}^{2}$, see Eq. (12), the damping integrals eventually read

$$
\begin{aligned}
& \chi_{1}=\oint\left\{\mathrm{b}_{\mathrm{RF}}-\mathcal{C}^{2} D_{x} \mathrm{~b}_{\delta x}-\left[\mathcal{S}_{-}^{2}-\mathcal{S}_{+}^{2}\right] D_{y} \mathrm{~b}_{\delta y}\right\} d s \\
& \chi_{2}=\oint\left\{\mathrm{b}_{\mathrm{RF}}-\left[\mathcal{S}_{-}^{2}-\mathcal{S}_{+}^{2}\right] D_{x} \mathrm{~b}_{\delta x}-\mathcal{C}^{2} D_{y} \mathrm{~b}_{\delta y}\right\} d s .
\end{aligned}
$$

Equations (26) and (27) are obtained from the diffusion and damping integrals through Eq. (B12).

Two limit cases are of interest. First, consider the case with a tune working point close to the sum resonance $(1,1)$ and betatron coupling such that $\left|f_{1001}\right| \ll\left|f_{1010}\right|$ anywhere in the ring. In this case $\mathcal{P} \rightarrow\left|f_{1010}\right|, \mathcal{S}_{-}^{2} \ll \mathcal{S}_{+}^{2}$, and $1=$ $\mathcal{C}^{2}-\mathcal{S}_{+}^{2}$. The two diffusion coefficients then simplify to

$$
\chi_{1}=\mathcal{C}^{2} \chi_{x}-\mathcal{S}_{+}^{2} \chi_{y} \quad \chi_{2}=-\mathcal{S}_{+}^{2} \chi_{x}+\mathcal{C}^{2} \chi_{y},
$$

where the uncoupled damping coefficients are defined as

$$
\begin{aligned}
& \chi_{x}=\oint\left[\mathrm{b}_{\mathrm{rf}}-D_{x} \mathrm{~b}_{\delta x}\right] d s, \\
& \chi_{y}=\oint\left[\mathrm{b}_{\mathrm{rf}}-D_{y} \mathrm{~b}_{\delta y}\right] d s .
\end{aligned}
$$

After expanding the RDT functions

$$
\begin{aligned}
& \mathcal{C}^{2}=\cosh \left(2\left|f_{1010}\right|\right), \\
& \mathcal{S}_{+}^{2}=\sinh \left(2\left|f_{1010}\right|\right),
\end{aligned}
$$

the damping coefficients may be written as

$$
\begin{gathered}
\chi_{1}=\cosh \left(2\left|f_{1010}\right|\right) \chi_{x}-\sinh \left(2\left|f_{1010}\right|\right) \chi_{y}, \\
\chi_{2}=-\sinh \left(2\left|f_{1010}\right|\right) \chi_{x}+\cosh \left(2\left|f_{1010}\right|\right) \chi_{y} .
\end{gathered}
$$

Second, consider the case with a tune working point close to the difference resonance $(1,-1)$ and betatron coupling such that $\left|f_{1010}\right| \ll\left|f_{1001}\right|$. In this case $\mathcal{P} \rightarrow i\left|f_{1001}\right|$,

\begin{tabular}{|c|c|c|c|c|c|}
\hline $\begin{array}{l}\left|C_{+}\right| \\
{[\%]}\end{array}$ & $\begin{array}{c}\left|C_{-}\right| \\
{[\%]}\end{array}$ & $\begin{array}{c}\mathcal{E}_{u} \\
\operatorname{MADX} \\
{[\mathrm{nm}]}\end{array}$ & $\begin{array}{c}\mathcal{E}_{u} \\
\text { Eq. (26) } \\
{[\mathrm{nm}]}\end{array}$ & $\begin{array}{c}\mathcal{E}_{v} \\
\text { MADX } \\
{[\mathrm{pm}]}\end{array}$ & $\begin{array}{c}\mathcal{E}_{v} \\
\text { Eq. }(27) \\
{[\mathrm{pm}]}\end{array}$ \\
\hline 0.24 & 0.07 & 4.03 & 3.95 & 0.738 & 0.733 \\
\hline 0.74 & 0.23 & 4.04 & 3.97 & 6.691 & 6.653 \\
\hline 1.23 & 0.39 & 4.06 & 3.99 & 18.86 & 18.79 \\
\hline 1.74 & 0.55 & 4.09 & 4.03 & 37.88 & 37.84 \\
\hline 2.24 & 0.73 & 4.15 & 4.08 & 64.87 & 65.04 \\
\hline
\end{tabular}
$\mathcal{S}_{+}^{2} \ll \mathcal{S}_{-}^{2}$, and $1=\mathcal{C}^{2}+\mathcal{S}_{-}^{2}$. The two diffusion coefficients in this case read

TABLE III. Comparison between the eigenemittance $\mathcal{E}_{u, v}$ computed by MADX and via Eqs. (26) and (27) for the ESRF storage ring lattice $\left(\mathcal{J}_{x} \simeq \mathcal{J}_{y}=1\right.$ and $\left.\mathcal{J}_{z} \simeq 2\right)$. Coupling is introduced by 32 skew quadrupoles and is quantified in the abscissas by the resonance stop bands $\left|C_{+}\right|$and $\left|C_{-}\right|$of Ref. [21].

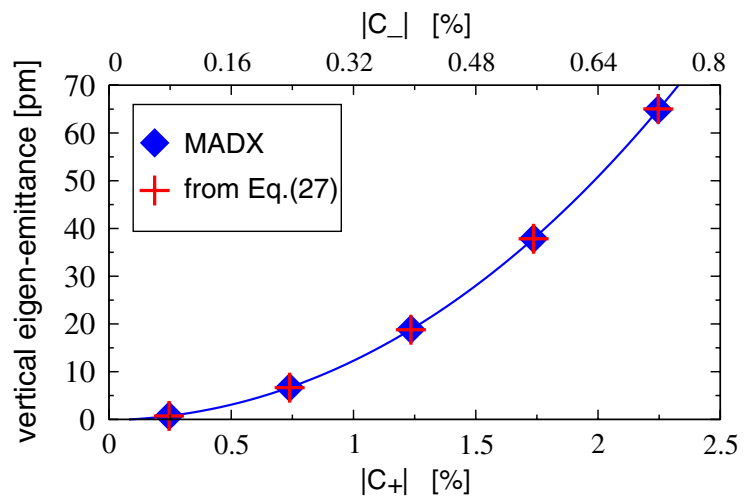

FIG. 12. Comparison between the vertical eigenemittance $\mathcal{E}_{v}$ computed by MADX (blue diamonds) and via Eqs. (26) and (27) (red plus) of Table III.

$$
\chi_{1}=\mathcal{C}^{2} \chi_{x}+\mathcal{S}_{-}^{2} \chi_{y} \quad \chi_{2}=\mathcal{S}_{-}^{2} \chi_{x}+\mathcal{C}^{2} \chi_{y}
$$

The RDT function instead becomes

$$
\begin{aligned}
& \mathcal{C}^{2}=\cos \left(2\left|f_{1001}\right|\right), \\
& \mathcal{S}_{-}^{2}=\sin \left(2\left|f_{1001}\right|\right),
\end{aligned}
$$

and the diffusion coefficients satisfy the following relations:

$$
\begin{aligned}
& \chi_{1}=\cos \left(2\left|f_{1001}\right|\right) \chi_{x}+\sin \left(2\left|f_{1001}\right|\right) \chi_{y} \\
& \chi_{2}=\sin \left(2\left|f_{1001}\right|\right) \chi_{x}+\cos \left(2\left|f_{1001}\right|\right) \chi_{y} .
\end{aligned}
$$

Equations (B34), (B43), (B44), (B48), and (B49) have been already derived in Ref. [8].

Equations (26) and (27) have been tested against MADX. In the first example the ESRF storage ring lattice is used. This has no focusing in the bending magnets $\left(\mathrm{b}_{\delta y}=0\right)$ and damping is by far dominated by the $\mathrm{rf}\left(\mathrm{b}_{\delta x} \ll \mathrm{b}_{\mathrm{rf}}\right)$, resulting in equal transverse partition numbers, $\mathcal{J}_{x} \simeq \mathcal{J}_{y}=1$ and $\mathcal{J}_{z} \simeq 2$. This test hence provides a verification of the coupling between the diffusion integrals only, i.e., the

TABLE IV. Comparison between the eigenemittance $\mathcal{E}_{u, v}$ computed by MADX and via Eqs. (26) and (27) for a lattice with strong quadrupolar fields in the main bending magnets $\left(\mathcal{J}_{x} \simeq 0.3, \mathcal{J}_{y}=1\right.$, and $\mathcal{J}_{z} \simeq 2.7$ ). Coupling is introduced by 40 skew quadrupoles and is quantified in the abscissas by the resonance stop bands $\left|C_{+}\right|$and $\left|C_{-}\right|$of Ref. [21].

\begin{tabular}{lccccc}
\hline \hline $\begin{array}{l}\left|C_{+}\right| \\
{\left[10^{-4}\right]}\end{array}$ & $\begin{array}{c}\mathcal{E}_{u} \\
{\left[1 C_{-} \mid\right.}\end{array}$ & $\begin{array}{c}\mathcal{E}_{u} \\
\text { MADX } \\
{[\mathrm{nm}]}\end{array}$ & $\begin{array}{c}\mathrm{Eq}_{v}(26) \\
{[\mathrm{nm}]}\end{array}$ & $\begin{array}{c}\text { MADX } \\
{[\mathrm{pm}]}\end{array}$ & $\begin{array}{c}\mathcal{E}_{v} \\
\text { Eq. (27) } \\
{[\mathrm{pm}]}\end{array}$ \\
\hline 0.8 & 9 & 1046 & 995 & 2.76 & 2.90 \\
2.5 & 2.6 & 1046 & 994 & 24.9 & 26.1 \\
4.1 & 4.3 & 1045 & 994 & 69.1 & 72.5 \\
5.8 & 6.0 & 1045 & 993 & 135 & 141 \\
7.4 & 7.7 & 1044 & 992 & 224 & 235 \\
\hline \hline
\end{tabular}




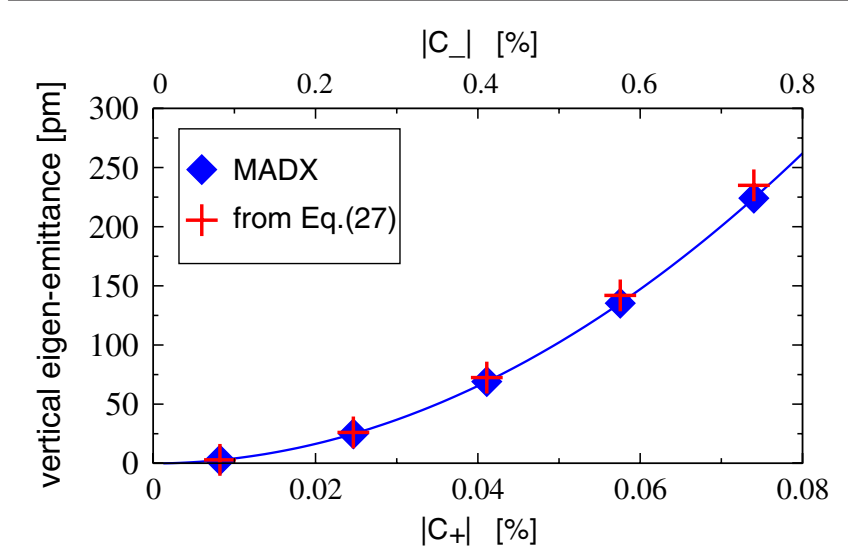

FIG. 13. Comparison between the vertical eigenemittance $\mathcal{E}_{v}$ computed by MADX (blue diamonds) and via Eqs. (26) and (27) (red plus) of Table IV.

numerators in Eqs. (26) and (27), both denominators being equal to $\oint \mathrm{b}_{\mathrm{rf}}(s) d s$. Results agree within $2 \%$ for $\mathcal{E}_{u}$ and within a fraction of percent for $\mathcal{E}_{v}$, as shown in Table III and plotted in Fig. 12.

The second test was performed by using a lattice with combined-function bending magnets, and hence with strong focusing inside the main dipoles. This results in $\mathrm{b}_{\delta x} \sim \mathrm{b}_{\mathrm{rf}}$ and in different partition numbers between the three planes, being $\mathcal{J}_{x} \simeq 0.3, \mathcal{J}_{y}=1$, and $\mathcal{J}_{z} \simeq 2$.7. This test hence provides a validation of coupling in both the diffusion and damping integrals. Results are reported in Table IV, with the values for the vertical emittance displayed in Fig. 13. The agreement is within 5\% for both emittances. The source of this larger discrepancy, as compared with the previous case, was not further investigated. The 5\% difference in the evaluation of the (large) horizontal eigenemittance may be transferred to the vertical one. The contribution of the oscillating terms in the damping and diffusion integrals, $R_{1}-R_{4}$, neglected in this derivation, may also be a source of discrepancy.

[1] Å. Andersson, M. Böge, A. Lüdeke, V. Schlott, and A. Streun, Nucl. Instrum. Methods Phys. Res., Sect. A 591593, 437 (2008).

[2] C. Thomas, G. Rehm, I. Martin, and R. Bartolini, Phys. Rev. ST Accel. Beams 13, 022805 (2010).

[3] R. Dowd, M. Boland, G. LeBlanc, and Y-R. E. Tan, Phys. Rev. ST Accel. Beams 14, 012804 (2011).

[4] M. Sands, SLAC Report No. SLAC-121, 1970.

[5] A. W. Chao, J. Appl. Phys. 50, 595 (1979).

[6] K. Ohmi, K. Hirata, and K. Oide, Phys. Rev. E 49, 751 (1994).
[7] H. Tanaka and A. Ando, in Proceedings of the Tamura Symposium on the Future of Accelerator Physics, Austin, Texas, AIP Conf. Proc. No. 356 (AIP, New York, 1996), p. 138.

[8] B. Nash, J. Wu, and A. W. Chao, Phys. Rev. ST Accel. Beams 9, 032801 (2006).

[9] B. Nash, Ph.D. thesis, Stanford University, USA [SLACR-820, 2006 (unpublished)].

[10] J. Wu, B. Nash, and A. Chao, in Proceedings of the 21st Particle Accelerator Conference, Knoxville, 2005 (IEEE, Piscataway, NJ, 2005), p. 1111.

[11] A. Terebilo, in Proceedings of the 19th Particle Accelerator Conference, Chicago, Illinois, 2001 (IEEE, Piscataway, NJ, 2001), p. 3203.

[12] W. Herr and F. Schmidt, CERN AB Report No. CERNAB-2004-027-ABP, 2004.

[13] C. Steier et al., in Proceedings of the 20th Particle Accelerator Conference, Portland, OR, 2003 (IEEE, New York, 2003), p. 3123.

[14] J. Safranek and S. Krinsky, Proceedings of the Orbit Correction and Analysis Workshop, AIP Conf. Proc. No. 315 (AIP, New York, 1994), pp. 163-169.

[15] K. Kubo et al., Phys. Rev. Lett. 88, 194801 (2002); Y. Honda et al., Phys. Rev. Lett. 92, 054802 (2004).

[16] R. Bartolini and F. Schmidt, Part. Accel. 59, 93 (1998).

[17] F. Zimmermann, K.L.F. Bane, T. Kotseroglou, J. Urakawa, H. Hayano, K. Kubo, T. Naito, K. Oide, N. Terunuma, N. Toge, T. Okugi, and S. Kashiwagi, SLAC Report No. SLAC/AP-113, 1998.

[18] R. Tomás García, Ph.D. thesis, University of Valencia, Spain [CERN-THESIS-2003-010 2003 (unpublished)].

[19] A. Franchi, Ph.D. thesis, J.W. Goethe University, Frankfurt am Main, Germany [GSI DISS 2006-07, 2006 (unpublished)].

[20] A. Franchi, E. Métral, and R. Tomás García, Phys. Rev. ST Accel. Beams 10, 064003 (2007).

[21] M. Minty and F. Zimmermann, Measurement and Control of Charged Particle Beams (Springer, Berlin, 2003).

[22] B. K. Scheidt and F. Epaud, in Proceedings of DIPAC09, Basel, Switzerland, 2009, edited by J. Chrin, M. Marx, V.R. W. Schaa, and V. Schlott, p. 50, http://accelconf .web.cern.ch/AccelConf/d09/papers/proceed.pdf.

[23] J. Safranek, Nucl. Instrum. Methods Phys. Res., Sect. A 388, 27 (1997).

[24] A. Ropert and L. Farvacque, in Proceedings of the 11th European Particle Accelerator Conference, Genoa, 2008 (EPS-AG, Genoa, Italy, 2008), p. 2106.

[25] B. K. Scheidt, in Proceedings of DIPAC07, edited by I. Andrian and V.R. W. Schaa (Venice, Italy, 2008), p. 72, http://accelconf.web.cern.ch/AccelConf/d07/papers/ tupb08.pdf.

[26] A. Franchi, R. Tomás García, and F. Schmidt, Phys. Rev. ST Accel. Beams 10, 074001 (2007).

[27] A. Bazzani, E. Todesco, G. Turchetti, and G. Servizi, Report No. CERN 94-02, 1994. 